\title{
Arbeit zur falschen Zeit am falschen Platz? Eine Matching-Analyse zu gesundheitlichen Beanspruchungen bei Schicht- und Nachtarbeit
}

\author{
Olaf Struck • Matthias Dütsch • Verena Liebig • \\ Angelina Springer
}

Angenommen: 21. März 2013 / Online publiziert: 10. April 2013

(C) Institut für Arbeitsmarkt- und Berufsforschung 2013

Zusammenfassung In vielen Untersuchungen werden von durch Schicht- und Nachtarbeit verursachte gesundheitliche Beeinträchtigungen berichtet. Im Regelfall lassen solche Studien soziodemographische Einflussfaktoren und Tätigkeitsmerkmale unberücksichtigt. Anhand der BIBB/BAuAErwerbstätigenbefragung 2006 wird im vorliegenden Aufsatz die Wirkung von zahlreichen tätigkeitsspezifischen und sozialstrukturellen Einflussfaktoren auf die physische und psychische Gesundheit sowie auf Schlafstörungen untersucht. Die Analyse erfolgt mit einem Matching-Ansatz. Dabei werden Schicht- und Nachtarbeitsgruppen mit möglichst strukturgleichen Kontrollgruppen in Bezug auf sozialstrukturelle Charakteristika und Tätigkeitsmerkmale verglichen. Dies ermöglicht es eigenständige Effekte der Lage der Arbeitszeit auf gesundheitliche Beeinträchtigungen zu extrahieren. Das Ergebnis: Bedeutsam für gesundheitliche Beanspruchungen ist vor allem, ob an körperlich, sozial oder psychisch belastetenden Arbeitsplätzen gearbeitet wird. Etwas weniger wichtig ist, ob dies am Tage oder in der Nacht geschieht.

O. Struck · M. Dütsch $(\varangle) \cdot$ V. Liebig

Professur für Arbeitswissenschaft, Otto-Friedrich-Universität

Bamberg, Feldkirchenstraße 21, 96052 Bamberg, Deutschland

e-mail: Matthias.Duetsch@uni-bamberg.de

O. Struck

e-mail: olaf.struck@uni-bamberg.de

url: http://www.uni-bamberg.de/arbeitswiss

V. Liebig

e-mail: Verena.Liebig@uni-bamberg.de

\section{A. Springer}

Institut für Arbeitsmarkt- und Berufsforschung (IAB)

der Bundesagentur für Arbeit (BA), Regensburger Straße 104,

90478 Nümberg, Deutschland

e-mail: Angelina.Springer@iab.de
Schlüsselwörter Nachtarbeit - Schichtarbeit · Gesundheit · Ergonomie $\cdot$ Arbeitsbelastung $\cdot$ Matching-Ansatz

Working at the wrong time at the wrong place? A matching-analysis on health stresses for shift and night work

\begin{abstract}
Many studies report diseases caused by shift and night work. As a rule, however, such studies do not take into account socio-demographic factors and job characteristics. Based on the BIBB/BAuA Employment Survey 2006, the present paper looks into the effects of various job-specific and socio-structural factors on physical and mental health as well as sleeping disorders. The analysis was carried out using the matching approach. Furthermore, the matching approach was used to form to shift and night work groups as structurally identical references as possible with regard to social-structural characteristics and job features. This strategy makes it possible to extract independent effects of the timing of work on health problems. The result: The question as to whether people work at physically, socially or psychologically strenuous workplaces is essential for their health stresses. It is slightly less important whether this work is done in the daytime or at night.
\end{abstract}

Keywords Night work · Shift work · Health · Ergonomics · Work stress · Matching approach

\section{Einleitung}

Etwa ein Viertel aller Beschäftigten in Deutschland arbeitet in Schichtarbeit, etwa ein Achtel arbeitet an 5 oder mehr Tagen im Monat in Nachtschichten (Beermann 2010). Entsprechend vieler Veröffentlichungen sind Schichtarbeit und ins- 
besondere Nachtarbeit eine wichtige Ursache für diverse gesundheitliche Gefährdungen. Beschrieben werden Wirkungen auf zirkadiane Rhythmus-Schlafstörung und auf Schlafmangel, Herz-Kreislauf- und Magen-Darm-Beschwerden sowie psychische Symptome. Doch handelt es sich dabei tatsächlich um einen kausalen Zusammenhang zwischen Schichtarbeit bzw. Nachtarbeit und gesundheitlichen Beeinträchtigungen? Oder sind es spezifische Merkmale, wie die Bedingungen am Arbeitsplatz bzw. ein niedriger sozialer Status, die gesundheitsgefährdend wirken und die charakteristisch für Beschäftigte in Schicht- bzw. Nachtarbeit sind, aber durchaus auch andere Beschäftigte kennzeichnen? Die Frage nach dem eigenständigen Erklärungsgehalt von Schicht- oder Nachtarbeit auf den gesundheitlichen Zustand soll im Folgenden mit Hilfe eines empirischen MatchingAnsatzes untersucht werden. Hierbei werden Beschäftigtengruppen analysiert, die sich in ihren soziodemographischen Charakteristika und ihren Tätigkeitsmerkmalen sehr stark ähneln, jedoch hinsichtlich ihres zeitlichen Arbeitseinsatzes unterscheiden. Der so ermittelte Einfluss von Schicht- und Nachtarbeit auf gesundheitliche Beanspruchungen wird anhand der BIBB/BAuA-Erwerbstätigenbefragung 2006 analysiert.

Der Beitrag erläutert im folgenden Abschn. 2 zunächst die Problemstellung anhand des Forschungsstandes. Nachfolgend werden der Datensatz, die Schätzmethode und die Modellannahmen beschrieben. Im Abschn. 4 werden die Ergebnisse der empirischen Analysen dargestellt und bewertet. Diese werden mit einem Matching-Ansatz berechnet. Er ist geeignet Selektionseffekte kontrolliert zu verringern. Es zeigt sich, dass Nacht- und Schichtarbeit einen vergleichsweise geringen eigenständigen Effekt auf die Gesundheit haben. Darüber hinaus verdeutlicht eine weitere Analyse, dass weniger die Lage der Arbeitszeit, sondern stärker die Belastungen an Arbeitsplätzen die Wahrscheinlichkeit gesundheitlicher Beschwerden erhöhen. Im letzten Abschnitt werden die Resultate knapp zusammengefasst.

\section{Problemstellung und Forschungsstand}

Schichtarbeit ist jede Form der Arbeitsgestaltung, die zu wechselnden Tag- und/oder Nachtzeiten oder konstant zu „,ungewöhnlich“ frühen oder späten Zeiten ausgeführt wird. Nach einem Zeitplan wechseln sich Arbeitnehmerinnen und Arbeitnehmer an einem Arbeitsplatz oder in einer übereinstimmenden Arbeitsaufgabe ab, wodurch lange und von der individuellen Arbeitszeit entkoppelte Betriebszeiten erreicht werden (BAG 1990). Nachtarbeit definieren wir entsprechend der Datengrundlage als Erwerbsarbeit zwischen 23 und 5 Uhr.

Nach den Daten der im vorliegenden Aufsatz analysierten BIBB/BAuA-Erwerbstätigenbefragung 2006 arbeiten 27,3\% aller abhängig Beschäftigten in Schichtarbeit und
$11,1 \%$ der Beschäftigten regelmäßig in Nachtarbeit zwischen 23 und 5 Uhr an 5 oder mehr Nächten im Monat. Für längere Betriebs-, Öffnungs- und Bereitschaftszeiten und damit für Schicht- oder Nachtarbeit gibt es gesellschaftliche, technologische und ökonomische Gründe. In den Bereichen Sicherheit, Gesundheit und Pflege sowie Kommunikation, Verkehrswesen und Energie ist es zum Schutz von Leben und Gesundheit oder zum Aufrechterhalt von Infrastrukturleistungen vielfach unumgänglich, dass Dienste rund um die Uhr angeboten werden. Hier ist der Anteil von Nachtschichtarbeit besonders hoch und sie gehört ebenso zum Berufsbild wie eine hohe Verantwortung am Arbeitsplatz. In Industriebetrieben sind es vor allem technologische und ökonomische Anlässe. Zum Teil benötigen Verfahrensprozesse, etwa in der chemischen Industrie, im Bau, in der Stahlproduktion und in Gießereien einen kontinuierlichen Arbeitseinsatz mit ebenfalls höheren Anteilen von Nachtschichtarbeit. Hinzu kommt, dass im Zuge verschärfter weltweiter Wettbewerbsbedingungen sowie bei hohen Investitionskosten in Maschinen und Anlagen, d.h. bei hohen fixen Kosten, die Unternehmen bestrebt sind, eine hohe zeitliche Kapazitätsauslastung zu erreichen, wobei in diesen Bereichen der Investitions- und Gebrauchsgüterproduktion sowie in der Nahrungs- und Genussmittelindustrie ein werktäglicher Zweischichtbetrieb häufiger anzutreffen ist als ein Dreischichtwechsel. Zweischichtarbeit steigert den Nutzungsgrad von Anlagekapazitäten, ohne dass deutlich steigende Lohnkosten anfallen, wie sie in Form von Zuschlägen vor allem bei Nacht- und Wochenendarbeit zu zahlen sind. Diese Zuschläge sind es dann auch, die auf Arbeitnehmerseite in diesen manuellen Berufen in der Industrie ein wesentliches Motiv für Schicht- und Nachtarbeit darstellen. Kundenbedürfnisse von unternehmerischen und privaten Akteuren, die ihrerseits häufig engen zeitlichen Restriktionen unterworfen sind, führten dazu, dass sich auch im Kundenservice, im technischen Support, in Handel und Logistik etc. die Verfügbarkeit rund um die Uhr ausgeweitet hat. Damit einhergehend wurden insbesondere in den wachsenden Dienstleistungsbereichen Tag-, Nacht- und Wochenendangebote ausgedehnt und die tägliche Erwerbsarbeitszeit zunehmend flexibilisiert. Allein Büro- und Beratungsberufe, etwa in Banken und Versicherungen blieben von dieser Entwicklung weitgehend ausgeklammert. Insgesamt hat sich der Anteil der in Schicht- und Nachtarbeit Tätigen in den letzten Jahrzehnten sukzessive erhöht (Seifert 2005).

Schichtarbeit und insbesondere Nachtarbeit gelten als Belastungen. Für die entsprechenden Beschäftigten werden drei zentrale Beanspruchungsprobleme diskutiert, die zum Teil miteinander im Zusammenhang stehen:

1. Versetzte Arbeits- und Freizeiten bereiten Schwierigkeiten die Teilhabe am sozialen Umfeld und dem familiären Leben zu organisieren (Beermann 2010; Estryn-Behar 
et al. 1990; Gadbois 1996; Knauth et al. 1983; Wedderburn 1975). Die daraus entstehenden psychischen Spannungen oder Versuche der Schlafanpassung an die ,normalen“ Rhythmen der sozialen Umwelt (Knauth et al. 1980; Folkard und Barton 1993), bei z.T. gleichzeitiger chronischer Müdigkeit, können sich dann wiederum auch negativ auf das gesundheitliche Gleichgewicht der Schichtarbeiter auswirken. Studien berichten von sozialem Stress bis hin zu Angstzuständen und Depressionen (Jamal 2004; Jansen et al. 2004; Pisarski et al. 2002). Die sozialen Beanspruchungen aufgrund sozialer Desynchronisation müssen aufgrund von Datenbeschränkungen im Folgenden weitgehend unberücksichtigt bleiben. Jedoch können die den sozialen Beanspruchungen vorgelagerten psychischen Gesundheitssymptome wie Nervosität und Reizbarkeit, Depressionen oder Burnout sowie Schlafstörungen betrachtet werden. Zudem werden Selektionseffekte aufgrund von häufiger berichteten Partnerschaftsproblemen in der Schichtarbeit durch das Kontrollgruppendesign, das anschließend näher beschrieben wird, minimiert, indem der Partnerschaftsstatus und die Frage nach Kindern im Haushalt berücksichtigt werden.

2. In vielen Studien wird vor allem gesundheitlichen Risiken wie Schlafproblemen, Magen- und Darmbeschwerden, Herz-Kreislauferkrankungen und psychischen Störungen eine besondere Bedeutung beigemessen. Ihre Ursachen stehen auch im Fokus der folgenden Analysen. Schichtarbeit und hier speziell Morgen- und Spätschichten sowie Nachtarbeit führen zu einer Desynchronisierung des Schlaf-Wachrhythmus und gehen häufig mit qualitativen und quantitativen Schlafstörungen einher (Hajak und Zulley 2008; Wedderburn 2000). Vielfach berichtet wird beispielsweise, dass Arbeitnehmer, die früh um 5:00 Uhr zur Morgenschicht antreten, nicht vor 22:00 Uhr zu Bett gehen und entsprechend Schlafdefizite aufbauen (Tilley et al. 1981; Knauth 1985; Folkard und Barton 1993; Kecklund und Åkerstedt 1995). Diese wiederum können zu vermehrter Schläfrigkeit (Hypersomnie) oder auch Nervosität führen. Mit vergleichbaren Effekten werden zudem für häufiger in Nachtarbeit Beschäftigte verkürzte Tagschlafdauern beobachtet, die auf die Desynchronisierung des zirkadianen Rhythmus und störende Lichtverhältnisse, Lärm und das soziale sowie familiäre Umfeld zurückgeführt werden (Ahasan et al. 2001; Åkerstedt und Gillberg 1981; Knauth 1985; Knauth et al. 1980; Knauth und Rutenfranz 1981; Van Cauter et al. 1994). Aufgrund der Desynchronisierung des Schlaf-Wachrhythmus finden sich Hinweise für psychische Störungen. Costa (1996) berichtet in einem Überblick über einzelne kleinere Branchenstudien zu Erwerbstätigen in Schichtarbeit und regelmäßiger Nachtarbeit von Angstzuständen, Depressionen und Neurosen (zu letzteren auch Bohle und Tilley 1989). Wirkungen von Schlafstörungen und Schlafmangel auf psychische Störungen, aber auch auf gastrointestinale und kardiovaskuläre Beschwerden werden als kurzfristige Wirkungszusammenhänge beschrieben, die langfristige und schwerwiegendere Folgen haben können. ${ }^{1}$

Gastrointestinale Beschwerden gelten in der Literatur nach den Schlafstörungen - als die zweithäufigsten Probleme infolge von Schicht- und insbesondere regelmäBiger Nachtarbeit (Angersbach et al. 1980; Wedderburn 2000). Costa (1996) spricht bei Nachtschichtarbeit von einem 2 bis 5-mal häufigeren Auftreten von Magenbeschwerden. Zudem werden Zusammenhänge zu Magen- und Zwölffingerdarmgeschwüren berichtet (Caruso et al. 2004; Knutsson 2003; Segawa et al. 1987). Als Ursache von Magen-Darmerkrankungen werden wiederum Störungen des Schlaf-Wachrhythmus angesehen. Dabei können diese Störungen Auswirkungen auf die Zeit der Aufnahme von Nahrung haben. Thematisiert werden Eile aufgrund eines verhinderten „,sozialen Essens“ im Familienkreis, ein eingeschränkter Zugang zu gesunder Nahrung oder der „Wunsch“ nach einem Nachtmahl zur Strukturierung der Nachtschicht etc. (Beermann 2010; Wedderburn 2000). Zudem können die Störungen die Wahl der Nahrung beeinflussen. Hier werden u. a. die Aufnahme ungeeigneter Mengen von schnellen „Sättigern“ in Form fettreicher und zuckerhaltiger Lebensmittel sowie der Genuss vermeintlicher Wachmacher wie Kaffee oder Tee genannt. Beide Effekte können dann die Verdauung bzw. Resorption von Nahrung negativ beeinflussen (ebd.). $\mathrm{Zu}$ berücksichtigen ist allerdings, dass insbesondere gastrologische Beschwerden, die sich frühzeitig schmerzhaft zur Geltung bringen, seit einigen Jahren sehr gut zu behandeln sind und entsprechend selten Einschränkungen für die Ausübung von Tätigkeiten bestehen (Costa 2000).

Auch für einen Zusammenhang zwischen kardiovaskulären Erkrankungen und Schicht- bzw. insbesondere Nachtarbeit bestehen teilweise Evidenzen (Bøggild und Knutsson

\footnotetext{
${ }^{1}$ In jüngerer Zeit wurde häufiger von einer positiven Korrelation von Nachtarbeit und Krebserkrankungen berichtet. Ende 2007 wurden Nacht- und Schichtarbeit in die Liste von wahrscheinlich krebserregender Agenzien der Internationalen Agentur für Krebsforschung aufgenommen (IARC 2007; Straif et al. 2007). Vermutet wird auf Basis von Tierexperimenten, dass ein geringer Melatoninspiegel durch Schlafstörungen und Schlafmangel möglicherweise das Immunsystem beeinträchtigt. In Überblicksstudien zur epidemiologischen Evidenz werden gesicherte Befunde u. a. aufgrund mangelnder Kontrolle von Tätigkeitsmerkmalen oder des Sozialstatus jedoch sehr kritisch gesehen (Costa et al. 2010; Kolstad 2008; Pukkala und Härmä 2007). So können beispielsweise für das Brustkrebsrisiko von Flugbegleiterinnen eher kanzerogene ionisierende Strahlungen als die Lage der Arbeitszeit verantwortlich gemacht werden. Für Brustkrebsrisiken von Krankenschwestern liegen etwas besser kontrollierte Studien vor (Schernhammer et al. 2006). Doch auch hier werden signifikante kausale Zusammenhänge bestritten (Kolstad et al. 2010; Pesch et al. 2010; Pronk et al. 2010). Da Krebserkrankungen im vorliegenden Datensatz nicht enthalten sind und darüber hinaus Langfristfolgen nicht untersucht werden können, müssen sie in dieser Untersuchung unberücksichtigt bleiben.
} 
1999; Knutsson 2003). Durch Schicht- und Nachtarbeit hervorgerufene neurovegetative Störungen führen teilweise zu hormonellen Reaktionen (insbesondere bei Katecholamine und Kortisol), wodurch wiederum negative Auswirkungen auf den Blutdruck, die Herzschlagfrequenz sowie auf thrombotische Lipid- und Glukosestoffwechselprozesse assoziiert sind (Van Amelsvoort et al. 2004; Karlsson et al. 2001). Berichtet werden zudem Wechselbeziehungen etwa zu psychomentalen Beanspruchungen oder zu Schlafstörungen hier insbesondere Insomnie (ebd.; Tenkanen et al. 1997; Wedderburn 2000). Große Längsschnittuntersuchungen bei Industriearbeitern (Tenkanen et al. 1997) und Krankenschwestern (Kawachi et al. 1995) zeigten, dass ein deutlich erhöhtes Risiko koronarer Herzerkrankungen auch bei Kontrolle etwa von Fettleibigkeit und Rauchen besteht. Allerdings finden sich immer wieder Ergebnisse, die nur eine sehr geringe Risikoerhöhung, etwa für Hypertonie bei den Schichtarbeitern ausweisen (Sakata et al. 2003). In einem neueren Studienüberblick sprechen Frost et al. (2009: 1) von einer ,limited epidemiological evidence for a causal relation between shift work and ischemic heart disease." In einer großen prospektiven bevölkerungsbasierten Kohortenstudie (Finnish Twin Cohort) fanden sich nach Berücksichtigung von soziodemografischen Determinanten und Lebensstilfaktoren keine Zusammenhänge zwischen Schichtarbeit und kardiovaskularen Erkrankungen (Hublin et al. 2010). Ebenso schreiben auch schon Bøggild et al. (1999) kardiologische Beschwerden dem häufig geringeren sozialen Status der Schichtarbeiter zu.

3. Teilweise wird darauf hingewiesen, dass in Schichtund Nachtarbeit Beschäftigte durch besondere körperliche und psychische Anforderungen am Arbeitsplatz anteilig in stärkerem Maße negativen Belastungen ausgesetzt sind als Personen, die im „normalen“, tageszeitlichen Rhythmus arbeiten (Bøggild et al. 2001; Beermann 2010; Knauth 1983). Zudem können auch arbeitsorganisatorische Faktoren und Arbeitsplatzmerkmale, wie etwa die Handlungsspielräume bei der Bewältigung von Arbeitsaufgaben, hohe Verantwortung und partiell große Stressbelastungen, die gesundheitliche Beanspruchung von Tag- und Nachtschichtgruppen beeinflussen (Badura und Feuerstein 1996: 283ff.). Diese arbeitsbedingten Belastungen treten z.B. bei Gesundheitsberufen oder im Polizeidienst auf, die anteilig häufig in Nachtschicht arbeiten, auch wenn für jeden einzelnen dieser letztegenannten Berufsgruppen deutlich weniger Nachtdienste im Monat anfallen als in der stärker durch Heteronomie gekennzeichneten industriellen Dreischichtarbeit.

Damit stellt sich grundsätzlich die Frage, ob nicht vor allem diese Bedingungen des Arbeitsplatzes und weniger die Lage der Arbeitszeit, zu der an belastenden Arbeitsplätzen gearbeitet wird, die zuvor genannten Gesundheitsgefährdungen erklären können (Costa 2000, 2004). Eigenständige oder moderierende Wirkungen von Charakteristika des Arbeitsplatzes sowie von Tätigkeitsmerkmalen sind allerdings in den bisherigen Studien zu gesundheitlichen Gefährdungen von Schicht- und Nachtarbeit weitgehend unberücksichtigt geblieben bzw. wurden aufgrund mangelnder Informationen in den Datensätzen nur unzureichend kontrolliert. Häufiger werden soziale Statusmerkmale einbezogen, während persönlichkeitsstrukturelle Merkmale (Selbstwirksamkeit, Kontrollkompetenz) oder andere Merkmale eines individuellen Beanspruchungsniveaus wiederum fehlen. Damit verbunden besteht eine sehr heterogene Befundlage, die eine Hypothesenbildung deutlich erschwert. Aus diesem Grund wird - in Anlehnung an regulative Belastungs-Beanspruchungskonzepte (etwa Hacker 2005) bzw. Anforderungs-Kontroll-Modelle (etwa Karasek und Theorell 1990) - in der vorliegenden explorativen Studie geprüft, ob bestimmte körperliche oder psychische Arbeitsbelastungen, die einerseits häufig Schicht- oder Nachtarbeit kennzeichnen, aber anderseits auch Merkmal bei Tagarbeit sind, einen stärkeren Einfluss auf die gesundheitliche Beeinträchtigung von Beschäftigten haben als die zeitliche Lage der Arbeitstätigkeit.

\section{Datengrundlage, Selektionsprobleme und Schätzmethode}

Im Folgenden werden zunächst der Datensatz beschrieben und danach wichtige (Unterscheidungs-)Merkmale von Schichtarbeit, Nachtarbeit sowie Nicht-Schichtarbeit vorgestellt. Dabei werden Selektivitätsprobleme im Vergleich der gruppenspezifischen Merkmale deutlich, so dass in einem weiteren Schritt der methodische Umgang damit zu erläutern ist. Am Ende des Abschnitts wird die ökonometrische Methode dargestellt.

\subsection{Datengrundlage}

Die empirischen Analysen werden mit den Daten der BIBB/BAuA-Erwerbstätigenbefragung 2006 durchgeführt (Hall et al. 2010; Hartmann 2006a). Es handelt sich um eine Repräsentativbefragung von ca. 20.000 Erwerbstätigen in Deutschland. ${ }^{2}$ Erhoben wurden neben soziodemographi-

\footnotetext{
${ }^{2}$ Dazu wurde gemäß dem Gabler-Häder-Verfahren eine adäquate Auswahlgrundlage zur Durchführung der telefonischen Befragung generiert. Die Auswahl der Zielpersonen erfolgte zweistufig. Zunächst wurde auf Basis eines Schichtungsmodells eine Telefonnummer und damit letztendlich ein Haushalt gezogen, um danach innerhalb des Haushalts über einen Zufallsmechanismus die Zielperson auszusuchen. Die entsprechende Auswahlgesamtheit wurde bei der BIBB/BAuAErwerbstätigenbefragung als die im Haushalt lebenden Personen ab 15 Jahren bestimmt. Aus dieser Auswahlgesamtheit wurde durch eine Zufallsauswahl mittels des Schwedenschlüssels die Zielperson ermittelt. Durch regelmäßige Strukturkontrollen der Stichprobe und eine darauf basierte gezielte Steuerung der Befragung wurde die Unterrepräsentation einzelner Zielgruppen ausgeglichen. Ausführlichere Informationen und eine Ausschöpfungsübersicht finden sich im Feldbericht bei Hartmann (2006a).
} 
schen und beruflichen Angaben u. a. zahlreiche Indikatoren zu Tätigkeitsmerkmalen bzw. physischen, psychischen und sozialen Anforderungen, Belastungen am Arbeitsplatz sowie Indikatoren zu gesundheitlichen Beeinträchtigungen (BIBB/BAuA 2006). Damit stellt die Befragung in großem Umfang Variablen für wesentliche Einflussfaktoren auf gesundheitliche Belastungen von Schicht- und Nachtarbeit bereit. Sie ist für quantitative Analysen in diesem Bereich derzeit alternativlos. Um befragungsbedingte Selektivitäten auszugleichen und die Repräsentativität zu gewährleisten, wurde auf Basis von Strukturmerkmalen eine Gewichtung am Mikrozensus 2005 gebildet (Hartmann 2006b). Diese Gewichtung wird in allen Berechnungen verwendet.

In der folgenden Analyse werden Vollzeitbeschäftigte im Alter zwischen 18 und 65 Jahren berücksichtigt. Auszubildende, Schüler und Studenten werden von der Analyse ausgenommen. Vollzeitbeschäftigung wird anhand einer Wochenarbeitszeit zwischen 35 und 50 Stunden definiert, um einerseits Belastungseffekte durch ein „Übermaß“ an Überstunden oder Nebenerwerbstätigkeiten zu umgehen sowie anderseits gute Regenerationsmöglichkeiten durch eine geringere Arbeitszeit auszuschließen. Unterschieden werden dabei vier Gruppen: (1) Nicht-Schichtarbeit, (2) Schichtarbeit mit Morgen- und/oder Abendschichten im Zeitraum von 5 bis $23 \mathrm{Uhr}$, (3) gelegentliche Nachtarbeit mit 1 bis 4 monatlichen Nachtarbeitsphasen in der Zeit zwischen 23 bis 5 Uhr sowie (4) häufige Nachtarbeit mit 5 oder mehr Nächten in dem vorher genannten Zeitraum. Die Dichotomisierung der Nachtarbeit an der Grenze von vier Nachtarbeitstagen im Monat basiert auf der Einschätzung, dass im Falle von Wechselschichtsystemen mit vier oder weniger Nachtarbeitstagen im Monat den entsprechenden Belastungen ausreichende Erholungsphasen gegenüberstehen. Mehr Nachtarbeitstage werden in der eingangs vorgestellten Literatur hingegen als besonders belastend eingeschätzt. In den ökonometrischen Analysen werden dann gesundheitliche Selbsteinschätzungen über schmerzhafte Beschwerden betrachtet, die ,während oder unmittelbar nach der Arbeit häufig auftreten“ (BIBB/BAuA 2006). Einbezogen werden häufig auftretende Schmerzen in bestimmten Teilen des Skeletts, im Magen-Darmbereich und im Herz-Kreislaufbereich sowie verschiedene psychische Belastungen und Schlafstörungen. Die jeweiligen Variablen sind mit 0 und 1 codiert, wobei 1 ein häufiges Auftreten der Symptome kennzeichnet.

\subsection{Selektionsprobleme}

Tabelle 1 präsentiert Deskriptionen ausgewählter Merkmale für die zuvor genannten vier Gruppen. Der Blick auf die Soziodemografie verdeutlicht zunächst, dass Schichtarbeit eher Frauenarbeit, Nachtarbeit hingegen eher Männerarbeit ist. Sie wird zudem etwas häufiger von Ausländern als von
Deutschen ausgeübt. Schichtarbeiter, aber auch Nachtarbeiter in 1 bis 4 Nächten im Monat sind etwas seltener verheiratet. Bis auf einen leicht höheren Anteil von Kindern im Haushalt bei Beschäftigten in Nachtarbeit mit 5 Nächten und mehr im Monat ist dieser Anteil in den anderen Gruppen in etwa gleich. Die Altersstruktur ist in allen Schicht- und Nachtarbeitsgruppen durchschnittlich etwas jünger. Unterschiede bestehen zudem hinsichtlich des höchsten Berufsabschlusses. Gut $8 \%$ der in häufiger Nachtarbeit Beschäftigten und knapp $14 \%$ der Schichtbeschäftigten haben keinen beruflichen Abschluss. Bei Personen in Tagarbeit beträgt dieser Anteil 6,4\%. Hingegen finden sich (Fach-) Hochschulabsolventen deutlich seltener in der Gruppe der Schichtbeschäftigten und in Nachtarbeit von 5 und mehr Nächten im Monat. Bezüglich des höchsten Bildungsabschlusses liegen keine Unterschiede zwischen Personen in Nachtarbeit zwischen 1 bis 4 Nächten und jenen in Tagarbeit vor.

Werden Tätigkeitmerkmale betrachtet, dann werden ebenfalls deutliche Differenzen sichtbar. ${ }^{3}$ Personen in Schichtarbeit sind vergleichsweise seltener, Erwerbstätige in Nachtarbeit zwischen 1 und 4 Nächten im Monat hingegen häufiger in verantwortungsvollen Tätigkeiten beschäftigt als Personen in Nicht-Schichtarbeit. Zudem ist der Leistungsdruck bei Nachtarbeitern in 1 bis 4 Nächten größer. Demgegenüber ist bei Schicht- und bei häufigerer Nachtarbeit vielfach die Monotonie höher und es bestehen öfters fachliche Fehlanpassungen. Insgesamt sind - wenngleich seltener bei Nachtarbeit zwischen 1 bis 4 Nächten - die Handlungsspielräume in Schicht- und Nachtarbeit geringer als bei NichtSchichtarbeit. Auch die körperlichen Belastungen, wie Arbeiten im Stehen, Tragen von Lasten, Arbeit in gebückter Haltung und mit Erschütterungen oder Gefahrenstoffen sowie unter Lärmeinfluss bzw. Belastung der Atemwege ist bei Schicht- und Nachtarbeit deutlich höher als bei Beschäftigten in Tagarbeit. Des Weiteren bestehen zwischen Wirtschaftszweigen deutliche Unterschiede. Hauptsächlich im produzierenden Gewerbe, im Gesundheitswesen sowie im Sektor Verkehr/Nachrichtenübermittlung sind die Anteile der Erwerbstätigen in Schichtarbeit sowie in Nachtschichtarbeit besonders hoch.

Damit zeigen die Deskriptionen, dass es systematische Differenzen in den Beschäftigtenstrukturen gibt. Erwerbstätige in Schicht- und Nachtarbeit zeichnet sich durch größere Anteile jüngerer, geringer qualifizierter und männlicher Arbeitnehmer aus. Diese Beschäftigten befinden sich

\footnotetext{
${ }^{3}$ Gefragt wurde, ob das jeweilige Merkmal bei der Arbeitstätigkeit „,häufig“, „manchmal“", „selten“ oder „nie“ vorkommt. Es handelt sich damit um strukturelle Merkmale der Arbeitstätigkeit, die hinsichtlich der Wahrnehmung nicht systematisch zwischen den jeweiligen (Nicht-)Schichtarbeitsgruppen variieren. Aus diesen Angaben wurden Dummy-Variablen gebildet, wobei der Wert 1 jeweils die Ausprägung „,häufig“" wiedergibt.
} 
Tab. 1 Deskriptionen für Schicht-, Nacht- und Nicht-Schichtarbeitsgruppen

\begin{tabular}{|c|c|c|c|c|}
\hline Merkmal & Nicht-Schichtarbeit & Schichtarbeit & Nachtarbeit 1 bis 4 Nächte & Nachtarbeit $\geq 5$ Nächte \\
\hline Frauen & 33,36 & $39,84(0,000)^{\mathrm{a}}$ & $24,82(0,000)^{\mathrm{a}}$ & $22,37(0,000)^{\mathrm{a}}$ \\
\hline Ausländische Staatsangehörigkeit & 08,11 & $11,03(0,001)$ & $08,85(0,504)$ & $11,62(0,000)$ \\
\hline Verheiratet & 56,30 & $50,80(0,000)$ & $51,92(0,029)$ & $54,69(0,272)$ \\
\hline Kinder im $\mathrm{HH}$ & 43,92 & $42,06(0,223)$ & $43,66(0,900)$ & $46,61(0,067)$ \\
\hline Alter $^{\mathrm{b}}$ & & $(0,000)$ & $(0,023)$ & $(0,000)$ \\
\hline Zwischen 18 und 35 Jahre alt & 32,54 & 40,40 & 37,15 & 39,90 \\
\hline Zwischen 36 und 50 Jahre alt & 44,88 & 43,49 & 43,76 & 45,40 \\
\hline Zwischen 51 und 65 Jahre alt & 22,58 & 16,11 & 19,09 & 20,80 \\
\hline Höchster Berufsabschluss ${ }^{\mathrm{b}}$ & & $(0,000)$ & $(0,864)$ & $(0,000)$ \\
\hline Keine Berufsausbildung & 6,41 & 13,97 & 7,20 & 8,15 \\
\hline Berufsausbildung & 59,47 & 75,00 & 58,59 & 77,92 \\
\hline Fachschule & 7,81 & 4,37 & 8,08 & 4,04 \\
\hline (Fach-)Hochschulabschluss & 26,32 & 6,67 & 26,14 & 9,88 \\
\hline \multicolumn{5}{|l|}{ Tätigkeitsmerkmale } \\
\hline Verantwortungsvolle Tätigkeit & 63,14 & $56,67(0,000)$ & $80,32(0,000)$ & $60,75(0,094)$ \\
\hline Häufiges Arbeiten unter Leistungsdruck & 57,11 & $56,35(0,616)$ & $67,11(0,000)$ & $56,71(0,784)$ \\
\hline Wenig Handlungsspielraum vorhanden & 18,96 & $34,15(0,000)$ & $26,14(0,000)$ & $40,48(0,000)$ \\
\hline Auftreten von Monotonie & 45,05 & $63,02(0,000)$ & $43,76(0,519)$ & $64,43(0,000)$ \\
\hline Häufig neue Aufgaben & 08,76 & $08,33(0,622)$ & $13,95(0,000)$ & $09,31(0,509)$ \\
\hline Fachliche Überforderung & 04,16 & $06,59(0,000)$ & $04,56(0,622)$ & $06,93(0,000)$ \\
\hline Fachliche Unterforderung & 11,20 & $15,87(0,000)$ & $13,82(0,041)$ & $17,69(0,000)$ \\
\hline Überforderung durch Arbeitspensum & 17,27 & $16,87(0,727)$ & $24,74(0,000)$ & $19,54(0,044)$ \\
\hline Unterforderung durch Arbeitspensum & 04,98 & $05,17(0,777)$ & $05,30(0,715)$ & $06,95(0,003)$ \\
\hline Häufiges Arbeiten im Stehen & 45,24 & $77,46(0,000)$ & $62,11(0,000)$ & $77,49(0,000)$ \\
\hline Häufiges Arbeiten im Sitzen & 62,24 & $30,95(0,000)$ & $55,07(0,000)$ & $35,50(0,000)$ \\
\hline Häufiges Tragen der Lasten & 19,93 & $33,49(0,000)$ & $29,52(0,000)$ & $32,64(0,000)$ \\
\hline Häufiges Arbeiten in gebückter Haltung & 13,46 & $18,63(0,000)$ & $19,24(0,000)$ & $16,59(0,002)$ \\
\hline Häufiges Arbeiten mit Erschütterungen & 03,83 & $05,24(0,020)$ & $06,75(0,000)$ & $11,54(0,000)$ \\
\hline Häufiges Arbeiten unter Belastung der Atemwege & 30,43 & $49,21(0,000)$ & $39,35(0,000)$ & $59,38(0,000)$ \\
\hline Häufiges Arbeiten mit Gefahrenstoffen & 05,56 & $09,21(0,000)$ & $15,44(0,000)$ & $15,30(0,000)$ \\
\hline Häufiges Arbeiten bei Lärm & 21,54 & $37,94(0,000)$ & $33,48(0,000)$ & $46,61(0,000)$ \\
\hline Häufiges Arbeiten mit Mikroorganismen & 03,95 & $10,84(0,000)$ & $15,56(0,000)$ & $11,99(0,000)$ \\
\hline Branchen $^{\mathrm{b}}$ & & $(0,000)$ & $(0,000)$ & $(0,000)$ \\
\hline Dienstleistung (ohne öffentliche) & 37,68 & 34,40 & 26,20 & 18,71 \\
\hline Baugewerbe & 10,41 & 2,30 & 5,09 & 1,87 \\
\hline Gesundheitswesen & 6,38 & 14,90 & 20,96 & 13,32 \\
\hline Land und Forstwirtschaft & 1,92 & 1,07 & 1,35 & 1,57 \\
\hline Produzierendes Gewerbe & 31,75 & 42,47 & 27,54 & 49,18 \\
\hline Verkehr & 1,27 & 2,06 & 5,99 & 7,63 \\
\hline Öffentliche Dienstleistung & 10,59 & 2,80 & 12,87 & 7,71 \\
\hline
\end{tabular}

${ }^{a}$ In den Klammern sind die $p$-Werte von $t$-Tests und Chi-Quadrat-Unabhängigkeitstests für Unterschiede zwischen den Beschäftigten in den jeweiligen Gruppen und den Nicht-Schichtarbeitern angegeben

${ }^{\mathrm{b}}$ Die Prozentangaben summieren sich aufgrund von Rundungsungenauigkeiten nicht auf 100

Quelle: BIBB/BAUA-Erwerbstätigenbefragung 2006, alle Angaben gewichtet, eigene Berechnungen 
eher in Arbeitsbereichen, die durch Monotonie und Heteronomie gekennzeichnet sind und die zudem häufig fachliche Fehlbeanspruchungen oder auch Überforderungen im Arbeitspensum sowie vielfach körperliche Belastungen aufweisen. Entsprechend lassen die deskriptiven Auswertungen bezüglich der Frage, ob eine Beschäftigung in Nachtund Schichtarbeit negative Auswirkungen auf die Gesundheit hat, Selektionseffekte vermuten, die zu berücksichtigen sind. ${ }^{4}$ Selektionsverzerrungen können beispielsweise dazu führen, dass ein beobachteter Gesundheitsunterschied zwischen Beschäftigten, die in Schichtarbeits- und in NichtSchichtarbeitssystemen tätig sind, nicht durch die versetzte Arbeitszeit bedingt ist, sondern die Ursache in belastenden Arbeitsplatz- und Tätigkeitsmerkmalen der Schichtarbeit zu suchen ist (Costa 2000, 2004; Siegrist und Dragano 2006; Thomas und Power 2010). Ebenso könnte es sein, dass Krankheitssymptome dieser Gruppen auf den durchschnittlich niedrigeren Ausbildungs- und Berufsstatus und damit z. T. einhergehende Risikofaktoren (etwa geringere gesundheitliche Prävention) zurückzuführen sind (Bøggild et al. 1999; Costa 2004; Jungbauer-Gans 2006; Mirowsky und Ross 2003), da ein niedriger sozialer Status mit einer externalen Kontrollüberzeugung sowie einem geringeren Wissen und einer verminderten Sensibilität für Gesundheit korreliert sind (Janßen 2001). Aus diesem Grund sind in den Untersuchungen Merkmale der Tätigkeit bzw. des Arbeitsplatzes und der beruflich-qualifikatorische Status sowie soziodemografische Charakteristika wie die Familiensituation, aber ebenso das Alter etc. zu kontrollieren.

\subsection{Schätzmethode}

Im Folgenden wird auf Basis eines ökonometrischen Schätzverfahrens eine systematische Drittvariablenkontrolle durchgeführt. Ein geeignetes statistischen Verfahren, um derartige beobachtbare Selektionsverzerrungen zu minimieren, ist das Propensity Score Matching (Rosenbaum und Rubin 1983). Der Matching-Ansatz folgt dem Prinzip einer „effects-of causes-Analyse" (Holland 1986) und ermöglicht es den Effekt der Schicht- und Nachtarbeit auf die gesundheitliche Verfassung zu isolieren. Die Bevorzugung des MatchingVerfahrens gegenüber einem multiplen Regressionsmodell wird in einem ersten Schritt in diesem Kapitel begründet. Dabei stellt das Matching ein nichtparametrisches Verfahren dar, für das im Gegensatz zu einer parametrischen Regression weniger Modellannahmen getroffen werden müssen und das somit robuster gegenüber Verzerrungen aufgrund von

\footnotetext{
${ }^{4}$ Diese Effekte sind auf der Basis von Querschnittdaten nur sehr schwer zu kontrollieren. Solange jedoch geeignete Längsschnittdaten fehlen, die wichtige Tätigkeitsmerkmale einschließen (Paridon et al. 2012: 99), muss bei Querschnittanalysen versucht werden Selektionseffekte zu minimieren.
}

Spezifikationsfehlern ist (Gangl und DiPrete 2006). Allerdings müssen in einem weiteren Schritt Überlegungen zu zwei zentralen Annahmen dieses nichtparametrischen Verfahrens angestellt werden, die erfüllt sein müssen, um die Kausalanalyse durchführen zu können.

Ein erster inhaltlicher Grund für die Nutzung des Matching-Verfahrens lässt sich aus der bestehenden Literatur ableiten. So gibt es Hinweise darauf, dass Schichtarbeiter Probleme mit dem Schlaf, der Verdauung usw. im Vergleich zu Tagarbeitern in höherem Maße als „natürlichen“ Bestandteil ihrer Arbeitssituation betrachten (Andersen 1970; Agervold 1976; Åkerstedt 1977). Hieraus kann der Schluss gezogen werden, dass Schichtarbeiter gesundheitliche Probleme anders wahrnehmen und seltener ansprechen als Tagarbeiter. Eine gesicherte empirische Evidenz ist hierfür nicht bekannt. Es ist jedoch zu bedenken, dass Beschäftigte in Schicht- und Nachtarbeit (ohne Kontrolle weiterer Variablen) zunächst einmal häufiger Beschwerden nennen als Erwerbstätige in Nicht-Schichtarbeit. Ein eventuell bestehender Verzerrungseffekt würde also nicht die Richtung eines Zusammenhangs verändern, er könnte aber die Stärke bzw. Signifikanzen von Zusammenhängen durchaus mindern. Deshalb werden, um eine derartige Selektivität zu kontrollieren, in der hier vorliegenden Analyse Personen in Schichtund Nachtarbeit mit einer Kontrollgruppe verglichen, die an vergleichbar belastenden Arbeitsplätzen beschäftigt sind und vergleichbare soziodemographische Merkmale aufweisen. Personen der Kontrollgruppe sollten dementsprechend einzelne gesundheitliche Probleme ebenfalls als „natürlichen" Bestandteil ihrer Arbeitssituation betrachten. In der vorliegenden Analyse wäre dann der zuvor beschriebene und möglicherweise existente „Verzerrungseffekt" kontrolliert.

Vergleichbares gilt für ein zweites wichtiges Selektionsmerkmal, den sogenannten Healthy-Worker-Effekt (Baillargeon 2001). Er kennzeichnet den Umstand, dass an besonders belastenden Arbeitsplätzen, wie u.a. in Nacht- und Schichtarbeit, vorwiegend Menschen arbeiten, die gesund und leistungsfähig sind, weil andere, die diesen Arbeitsbedingungen nicht oder nicht in ausreichendem Maße gewachsen sind, diese Tätigkeit entweder gar nicht erst auf sich nehmen (dürfen) oder bei gesundheitlichen Beeinträchtigungen aufgeben (Angersbach et al. 1980; Wedderburn 2000). Aus drei Gründen kann im Folgenden ein Healthy-Worker-Effekt weitgehend ausgeschlossen werden. Zum einen können wir davon ausgehen, dass - sofern erkrankte „Aussteiger“ in geringer belastende Tätigkeiten in Nicht-Schichtarbeit integriert werden können - mit der Arbeitszeitlage zusammenhängende gesundheitliche Symptome zeitnah abklingen. ${ }^{5}$ Zum anderen unterstützt der Abgleich der von der Lage der

\footnotetext{
${ }^{5}$ In den zugrunde gelegten Daten wurden während oder direkt nach der Arbeit auftretende Symptome erfragt.
} 
Arbeitszeit unabhängigen Arbeitsplatzmerkmale und der soziodemografischen Charakteristika bei der Bildung der statistischen Zwillingen ebenfalls die Kontrolle dieses Effektes, da Schichtarbeiter, die aus gesundheitlichen Gründen stark beanspruchende Arbeitsplätze verlassen, in der Regel nicht auf gleichermaßen durch Belastungsfaktoren gekennzeichnete Nicht-Schichtarbeitsplätze wechseln werden. Dadurch wird die mögliche Verzerrung des Effektes der Tagarbeit, die auf die gesundheitlich beeinträchtigten, ehemaligen Schichtarbeiter zurückzuführen ist, minimiert. Da es sich des Weiteren bei den aus gesundheitlichen Gründen aus Schicht- oder Nachtarbeit aussteigenden Personen um vergleichsweise ältere Beschäftigte handelt, die zudem eine längere Berufserfahrung haben, wird der Healthy-WorkerEffekt auch durch die vergleichbare Altersverteilung und Berufserfahrung in den Vergleichsgruppen gemindert.

In Zusammenhang mit den beiden inhaltlichen Gründen steht der methodische Vorteil des Matchings, dass die Qualität des Abgleichs der Schicht- und Nachtarbeitsgruppen mit der jeweiligen Kontrollgruppe betrachtet und eingeschätzt werden kann. Dadurch wird gewährleistet, dass mit Hilfe des experimentellen Designs tatsächlich vergleichbare „statistische Zwillinge" gebildet werden.

Bevor das Matching-Verfahren in den nachfolgenden Analysen angewendet wird, muss noch die Plausibilität zweier zentraler Annahmen geprüft werden. So besteht eine Voraussetzung kausalen Schließens darin, dass die $\mathrm{Zu}$ ordnung von Personen zur Experimental- sowie zur Kontrollgruppe zufällig geschieht, so dass sich die Gruppen in ihren Charakteristika nicht unterscheiden und auch keine Selbstselektion der Personen in eine der Gruppen erfolgt. Bei der Analyse der vorliegenden Befragungsdaten kann jedoch nicht, wie in der klassischen Experimentalforschung, eine zufällige Zuweisung zur Gruppe der Schicht- bzw. Nachtarbeiter sowie der Tagarbeiter angenommen werden. Der Voraussetzung der ,conditional independence assumption" (CIA) kann aber dadurch Rechnung getragen werden, dass zur Bildung der Vergleichsgruppen Kovariaten ausgewählt werden, die unbeeinflusst von den Ausprägungen der Ergebnisvariablen sind. Zudem darf keine Selbstselektion der Beschäftigten in die jeweiligen Gruppen aufgrund des Gesundheitszustandes stattfinden (Gangl und DiPrete 2006; Rosenbaum und Rubin 1983).

Die „conditional independence assumption“ sehen wir als erfüllt an, da zum einen der vorliegende Datensatz eine große Bandbreite an sozio-demografischen Variablen sowie Angaben zum Alter und zu den Arbeitsplatz- und Tätigkeitsmerkmalen enthält, welche gemeinsam die Beschäftigung in Schicht- und Nachtarbeit sowie die gesundheitliche Verfassung umfassend erklären (Beermann 2010; Gadbois 1996; Knauth 1983). Darüber hinaus werden Beschäftigte, die eine anerkannte Behinderung haben, aus dem Untersuchungssample ausgeschlossen, da schwerbehinderte Arbeitnehmer ihren Anspruch auf eine behinderungsgerechte Gestaltung der Arbeitszeit mit der Maßgabe einer Freistellung von Schicht- oder Nachtarbeit gegenüber dem Arbeitgeber geltend machen können (SGB 9 § 81). Damit sollte durch die Auswahl geeigneter Kovariaten und durch die Dateneingrenzung keine Zuweisung zur Schicht- bzw. Nachtarbeitsgruppe oder Kontrollgruppe auf Basis der gesundheitlichen Verfassung stattfinden.

Eine zweite Annahme stellt die ,stable unit-treatment value assumption“ (SUTVA) dar (Gangl und DiPrete 2006; Rosenbaum und Rubin 1983). Diese besagt, dass ein durch die Schicht- bzw. Nachtarbeit bedingter Einheitseffekt bestehen muss. Somit dürfen keine Folgewirkungen aufgrund sozialer Interaktionen zwischen den Schicht- bzw. Nachtarbeitsgruppen und den Kontrollpersonen wegen der Häufigkeit und Verteilung sowie aufgrund von Gleichgewichtseffekten von Schicht- oder Nachtarbeit vorliegen. In den folgenden Analysen werden mögliche ,spill-over“ Probleme, wie die Überlegungen zum Healthy-Worker-Effekt zeigen, weitestgehend vermieden. Gleichwohl können solche Effekte aufgrund von Datenrestriktionen nicht vollständig ausgeschlossen werden, so dass dann die Effekte der Schichtund Nachtarbeit auf die gesundheitliche Verfassung tendenziell unterschätzt werden. ${ }^{6}$ Des Weiteren sollten Einheitseffekte sowohl für die Nachtarbeit als auch für Schichtarbeit im Zeitraum zwischen 5 und 23 Uhr vorliegen, da in Deutschland das sogenannte europäische Modell von Wechselschichtsystemen von zentraler Bedeutung ist (Seifert 2005).

\section{Empirische Analysen}

In diesem Kapitel werden zunächst die Ergebnisse der Logit-Schätzungen und die Matching-Qualität betrachtet. Anschließend erfolgen die Analysen zur gesundheitlichen Gefährdung durch Schicht- und die beiden Arten der Nachtarbeit. $^{7}$

\footnotetext{
${ }^{6}$ Das ,spill-over“ Problem kann mit Längsschnittdaten, sofern diese geeignete Variablen enthalten, besser behoben werden. Allerdings existiert bislang keine entsprechende Datenbasis zur Bearbeitung der vorliegenden Fragestellung.

${ }^{7}$ Auch zur Berechnung der Propensity Scores wurden die Surveygewichte (siehe Abschn. 3.1) herangezogen. Dabei gibt es unseres Wissens in der bisherigen Literatur keine eindeutigen Aussagen, ob im Rahmen des Propensity Score Matchings Gewichte verwendet werden sollten. Wir haben uns - wie von Leuven und Sianesi (2003) vorgeschlagen - dafür entschieden, die Propensity Scores sowohl mit als auch ohne Gewichtung zu berechnen und zu prüfen, in welchem Fall das Balancing der Kovariaten besser gelingt. Aufgrund des besseren Balancings haben wir in den Berechnungen die Gewichte genutzt.
} 


\subsection{Ergebnisse der Logit-Schätzungen}

Das Matching wird auf Basis von Propensity Scores, die auf Selektionsmodellen beruhen, durchgeführt. Dazu werden Logit-Modelle geschätzt, welche die jeweiligen Wahrscheinlichkeiten wiedergeben, Schichtarbeit im Zeitraum zwischen 5 und 23 Uhr oder zweitens in 1 bis 4 Nächten im Monat bzw. drittens in 5 oder mehr Nächten im Monat zu arbeiten. ${ }^{8}$ Die entsprechenden Dummy-Variablen nehmen den Wert 0 für Tagarbeiter an, der Wert 1 ist jeweils den Schicht- und den zwei Nachtarbeitsgruppen zugeordnet. In das Selektionsmodell müssen relevante Kovariaten aufgenommen werden, welche die Zugehörigkeit zur Gruppe der Schicht- bzw. Nachtarbeiter erklären und deren Verteilung für die weitere Kausalanalyse angeglichen wird. Dabei muss unter Kontrolle der Kovariaten die konditionale Unabhängigkeit von der Beschäftigung in Schicht- bzw. Nachtarbeit und den Angaben zur gesundheitlichen Verfassung gewährleistet sein. Bei der Auswahl der Kovariaten orientieren wir uns an bisherigen Erkenntnissen aus der arbeitswissenschaftlichen, gesundheitssoziologischen und medizinischen Forschung sowie entsprechenden Theorien. Als Kovariaten gehen Angaben zu Alter, Geschlecht und Familienstand, Kindern im Haushalt und Staatsangehörigkeit sowie zu dem höchsten Berufsabschluss in die Selektionsmodelle ein. Strukturelle Bedingungen am Arbeitsplatz werden anhand von vielfältigen Variablen abgebildet, die beispielsweise Informationen darüber beinhalten, ob an einem Arbeitsplatz verantwortungsvolle Tätigkeiten ausgeführt werden oder die Arbeiten häufig unter Leistungsdruck, häufig im Stehen etc. verrichtet werden. Darüber hinaus werden sieben Branchen differenziert. Diese sozial- und arbeitsplatzstrukturellen Merkmale erfüllen die ,,conditional independence assumption“ (CIA) und hängen nicht von der Beschäftigung in Schicht-, Nacht- oder Nicht-Schichtarbeit ab.

Das so spezifizierte Logit-Modell für die Schichtarbeit erreicht ein Pseudo- $R^{2}$ von 17,9 \%, wobei 86,23 \% der Fälle korrekt klassifiziert werden. ${ }^{9}$ Die Schätzung für 1 bis 4 Nachtschichten im Monat erzielt ein Pseudo- $R^{2}$ von $12,0 \%$ bei 90,64 \% richtig klassifizierten Fällen, während das Modell zur Nachtarbeit in 5 oder mehr Nächten im Monat ein Pseudo- $R^{2}$ von $24,3 \%$ und 86,38 \% korrekt klassifizierte Fälle ausweist. Die Modellgüte wurde zudem mit dem Hosmer-Lemeshow (HL) Test überprüft. Dazu wurden zehn Subgruppen (,deciles of risk“) gebildet, um die vorhergesagten und beobachteten Werten zu vergleichen. Unter Verwendung von $\mathrm{df}=8$ Freiheitsgraden lässt sich für das Modell zur Schichtarbeit ein $\chi^{2}$-Wert von 6,33 ( $\left.p=0,601\right)$, für

\footnotetext{
${ }^{8}$ Die Umsetzung erfolgt mit dem PSCORE-Programm von Becker und Ichino (2002) sowie dem PSMATCH2-Verfahren von Leuven und Sianesi (2003) mit der Statistik-Software Stata.

${ }^{9}$ Als cutoff wurde jeweils der Wert $p=0,5$ herangezogen.
}

Nachtarbeit in 1 bis 4 Nächten im Monat ein $\chi^{2}$-Wert von 3,17 ( $p=0,923$ ) und für Nachtarbeit in 5 oder mehr Nächten im Monat ein $\chi^{2}$-Wert von 9,14 $(p=0,331)$ ermitteln. Somit kann in allen Fällen die Nullhypothese, dass alle Differenzen zwischen den geschätzten und beobachteten Werten gleich Null sind, aufrechterhalten werden. Darüber hinaus wurde eine Regressionsdiagnostik bezüglich Multikollinearität mit Hilfe des ,Variance Inflation Factor“ (VIF) durchgeführt. Dieser bietet ein Maß dafür, wie stark die Kovariaten untereinander korrelieren. Die Werte des VIF variieren für die Kovariaten des Modells für Schichtarbeit zwischen 1,06 und 3,55, bei dem Modell zur Nachtarbeit in 1 bis 4 Nächten im Monat zwischen 1,07 und 3,87 sowie für die Nachtarbeit in 5 oder mehr Nächten im Monat zwischen 1,09 und 3,57. Damit führen die einbezogenen Kovariaten zu keinem Multikollinearitätsproblem, da alle Werte des Variance Inflation Factors unter dem Schwellenwert von 5 liegen (Urban und Mayerl 2006). Insgesamt weisen die angeführten Kennzahlen und Tests auf eine gute Modellgüte hin. Im Folgenden werden die Ergebnisse der Logit-Schätzungen vorgestellt.

Die multivariaten Analysen ${ }^{10}$ zeigen, dass Frauen hoch signifikant häufiger Schichtarbeit und Männer hingegen höchst signifikant häufiger Nachtarbeit leisten. Beim Partnerschaftsstatus und der Frage nach Kindern im Haushalt sowie bei der Staatsangehörigkeit bestehen keine signifikanten Gruppenunterschiede. Beschäftigte, die über 50 Jahre alt sind, befinden sich deutlich seltener in Schicht- und Nachtarbeit in mehr als 4 Nächten im Monat. Die Wahrscheinlichkeit 1 bis 4 Nachtschichten im Monat zu arbeiten ist demgegenüber altersunabhängig. Eine „Besonderheit“ bei Nachtarbeit von 1 bis 4 Nächten im Monat zeigt sich auch bei der Betrachtung des Berufsabschlusses. Anders als in den beiden anderen, zeitlich versetzten Arbeitsformen, entspricht hier der Anteil der Hochschulabsolventen dem Anteil in der Kontrollgruppe der Nicht-Schichtarbeit. Dieser Effekt wird in dieser Nachtarbeitsgruppe vor allem durch Ärzte im Gesundheitswesen hervorgerufen, die durch eine höhere Verantwortung, Weiterbildungsteilnahmen und Arbeiten unter Leistungsdruck gekennzeichnet sind. ${ }^{11}$ Beschäftigte in der Schichtarbeit und in der Nachtarbeit in 5 oder mehr Nächten im Monat besitzen demgegenüber anteilig deutlich seltener höhere Berufsabschlüsse. Im Vergleich der Wirtschaftszweige ist die Wahrscheinlichkeit, in Schicht- oder Nachtarbeit tätig zu sein, erwartungsgemäß in den Sektoren Verkehr/Nachrichtenübermittlung und Gesundheit besonders groß.

\footnotetext{
${ }^{10}$ Die Resultate dieser Logit-Schätzungen sind in den Tab. 4, 5, 6 im Anhang dokumentiert.

${ }^{11}$ Die Aussagen zu Wirtschaftszweigen basieren auf gesonderten multivariaten Logit-Modellen zu einzelnen Wirtschaftszweigen. Diese können aus Platzgründen nicht dokumentiert werden.
} 
Bei den Arbeitsplatz- und Tätigkeitsmerkmalen zeigt sich wiederum der „Sondereffekt“ der Ärzte in der Gruppe der Nachtarbeit bis zu 4 Nächten im Monat. Die Tätigkeiten sind deutlich überdurchschnittlich durch Arbeit mit Mikroorganismen und Gefahrenstoffen sowie durch hohe Verantwortung charakterisiert. Demgegenüber können geringe Handlungsspielräume, Monotonie, häufiges Arbeiten im Stehen und bei Lärm vor allem in der Gruppe der Schichtarbeit und der Nachtarbeit in 5 oder mehr Nächten im Monat beobachtet werden. Bei der Nachtarbeit in 5 und mehr Nächten kommen dann noch häufiges Arbeiten mit Erschütterungen, Belastungen der Atemwege und ebenfalls Arbeiten mit Mikroorganismen hinzu. Die noch in den oben vorgestellten deskriptiven Ergebnissen sichtbaren Risiken des Tragens von Lasten sowie fachliche Über- oder Unterforderungen in der Schicht- bzw. Nachtarbeit sind in diesen multivariaten Modellen insignifikant.

\subsection{Auswahl des Matchingalgorithmus und Beschreibungen der Matching-Qualität}

Die mit Hilfe des Selektionsmodells ermittelten Propensity Scores werden nun herangezogen, um die Kontrollgruppen zu bilden. Dies kann mit verschiedenen Zuordnungsalgorithmen bewerkstelligt werden. ${ }^{12}$ In den nachfolgenden Analysen wird ein Caliper Matching in Kombination mit einem Nearest-Neighbor-Verfahren gewählt. Dabei greifen wir auf ein Oversamling von bis zu 5-Nearest-Neighbors zurück, um jedem Schichtarbeiter und jedem Nachtarbeiter aus der großen potenziellen Kontrollgruppe maximal fünf möglichst strukturgleiche Nicht-Schichtarbeiter zuzuordnen. Durch das Oversampling wird die Varianz der Parameterschätzungen und die Anfälligkeit hinsichtlich möglicher irrelevanter Parameter verringert (Caliendo und Kopeinig 2008; Gangl und DiPrete 2006). Das Matching wird mit einer sehr engen maximal zulässigen Abweichungsdistanz $\Psi$ von 0.001 durchgeführt, um einen möglichst guten Abgleich aller beobachteten Kovariaten gewährleisten und eine hohe Biasreduktion erreichen zu können (Dehejia und Wahba 2002). ${ }^{13}$

\footnotetext{
${ }^{12}$ Einen Überblick über die Zuordnungsalgorithmen bieten Caliendo und Kopeinig (2008: 41f.).

${ }^{13}$ Die Sensitivität der Ergebnisse in Bezug auf das gewählte Matching-Verfahren wurde ausführlich getestet. In die Analyse wurden 1-Nearest-Neighbor-Verfahren mit und ohne Zurücklegen sowie 5und 10-NN-, Kernel- und Radius-Prozeduren jeweils ohne maximale Abweichungsdistanzen sowie mit den Werten $\Psi=0.01, \Psi=0.0005$ und $\Psi=0.0001$ einbezogen. Es hat sich zunächst gezeigt, dass sich hinreichend ähnliche Vergleichspersonen in den jeweiligen Gruppen befinden, die ein Oversampling ermöglichen. Die Auswahl der Abweichungsdistanz basiert auf der Feststellung, dass ab einem Caliper von 0.0005 relativ viele gute bzw. sehr gute Vergleichspersonen ausfallen, so dass dann für einen Teil der Schicht- bzw. Nachtarbeiter keine hinreichend ähnlichen Kontrollpersonen gefunden werden konnten.
}

Die Güte des Matchings ist für die Aussagekraft der generierten Ergebnisse entscheidend (Dehejia und Wahba 2002). Dazu müssen in einem für das Untersuchungsziel informativen Datensatz ausreichend viele Personen zur Bildung einer Kontrollgruppe vorhanden sein, die den beobachteten Schicht- und Nachtarbeitsbeschäftigten in ihren soziodemografischen und tätigkeitsspezifischen Charakteristika ähnlich sind, damit beide Gruppen einen gemeinsamen Stützbereich haben und die „,common support“Bedingung erfüllt ist (Caliendo und Kopeinig 2008). Nach der Datenaufbereitung bzw. vor dem Matching stehen 1.047 Vollzeiterwerbstätige Schichtarbeiter, 702 Vollzeiterwerbstätige Nachtarbeiter zwischen monatlich 1 und 4 Nächten, 1.168 Vollzeiterwerbstätige Nachtarbeiter mit monatlich 5 und mehr Nächten und 6.472 Vollzeiterwerbstätige ohne Schichtarbeit zur Verfügung. Die große Fallzahl der zuletzt genannten Gruppe, aus der die Kontrollpersonen gewonnen werden, schafft die Grundlage für eine gute Matching-Qualität. Abbildung 1 im Anhang zeigt Kern-Dichte-Schätzer der Verteilungen der Propensity Scores in den Schicht- und Nachtarbeits- sowie den NichtSchichtarbeitsgruppen vor dem Matching. Insbesondere die Verteilungen der Schichtarbeiter sowie Nachtarbeiter in 5 oder mehr Nächten und der Vergleichsgruppe sind deutlich gegeneinander verschoben. Allerdings überschneiden sich die Propensity Scores $P(X)$ der Schichtarbeiter und der Kontrollgruppe im Bereich $0<P(X)<0$,6, die der Nachtarbeiter zwischen 1 und 4 Nächten und der Vergleichsgruppe im Bereich $0<P(X)<0,4$ sowie der Nachtarbeiter mit 5 Nächten oder mehr und der Nicht-Schichtarbeiter im Bereich $0<P(X)<0,8$, so dass ein hinreichend großes Potential zur Bildung statistischer Zwillinge vorhanden ist (Caliendo und Kopeinig 2008; Gangl und DiPrete 2006).

Zur Beurteilung der Qualität des Matchings stehen verschiedene statistische Kennzahlen und Tests zur Verfügung, anhand derer festgestellt werden kann, ob durch das Matching ein zufriedenstellender Abgleich der Personen in Schicht- bzw. Nachtarbeit und der Kontrollpersonen hinsichtlich der beobachtbaren Variablen stattgefunden hat (Caliendo und Kopeinig 2008: 47f.). Die entsprechenden Balancing-Tests sind in den Tab. 7 bis 9 im Anhang dokumentiert. Ein wichtiges Maß stellt die standardisierte Verzerrung $\left(\mathrm{SB}^{14}\right)$ der Kovariaten dar. Der SB ist vor dem Matching definiert als Prozentwert, der sich aus der Differenz der Gruppenmittelwerte von Schicht- bzw. Nachtarbeitern sowie Nicht-Schichtarbeitern dividiert durch die Wurzel aus dem Durchschnitt der Varianzen der jeweiligen Gruppen ergibt:

$\mathrm{SB}_{\text {vorher }}=100 \times \frac{\bar{X}_{1}-\bar{X}_{0}}{\sqrt{0,5 \times\left(V_{1}(X)+V_{0}(X)\right)}}$

${ }^{14}$ Die Abkürzung SB steht für standardized bias. 
Nach dem Matching wird die Differenz zwischen den Mittelwerten der Kovariaten der jeweiligen Schicht- und Nachtarbeitsbeschäftigten $\left(\bar{X}_{1 M}\right)$ und der gematchten Beschäftigten ohne Schichtarbeit $\left(\bar{X}_{0 M}\right)$ berechnet und ins Verhältnis zur Wurzel aus dem Durchschnitt der Varianzen in den Gruppen gesetzt.

$\mathrm{SB}_{\text {nachher }}=100 \times \frac{\bar{X}_{1 M}-\bar{X}_{0 M}}{\sqrt{0,5 \times\left(V_{1 M}(X)+V_{0 M}(X)\right)}}$

Damit veranschaulichen die standardisierten Verzerrungen, ob die Unterschiede zwischen den Beschäftigten in Schicht- bzw. Nachtarbeit und den Personen in NichtSchichtarbeit im Hinblick auf die Einflussgrößen durch das Matching hinreichend minimiert werden konnten. Zur objektiven Bewertung der Verbesserung der Verzerrung gibt es keinen Test. Jedoch merken Caliendo und Kopeinig (2008: 48) an, dass in den meisten empirischen Studien eine Reduktion der standardisierten Verzerrung auf einen Wert unter $5 \%$ als ausreichend angesehen wird. Der SB von Nicht-Schichtarbeit und Schichtarbeit nimmt vor dem Matching einen Wert von 22,9\% und nach dem Matching von 2,7 \% an. Die entsprechenden Prozentwerte für Nicht-Schichtarbeit und Nachtarbeit bis zu 4 Nächten im Monat betragen 14,9\% vor und 3,0 \% nach dem Matching. Für Nicht-Schichtarbeit und Nachtarbeit von 5 Nächten und mehr im Monat wird eine standardisierte Verzerrung von 26,3\% vor und 4,2\% nach dem Matching berechnet. Folglich konnte der SB durch alle drei Matching-Prozeduren hinreichend verringert werden.

Ein weiterer Ansatz zur Prüfung der Matching-Qualität greift auf $t$-Tests zurück, um die Mittelwerte der Kovariaten vor und nach dem Matching zu vergleichen (Caliendo und Kopeinig 2008: 48f.). Die Tab. 7 bis 9 im Anhang zeigen, dass sich vor dem Matching sehr viele Charakteristika zwischen der Nicht-Schichtarbeit und der Schichtarbeit bzw. den beiden Varianten der Nachtarbeit signifikant unterscheiden. Hingegen existieren nach dem Matching, wie von Dehejia und Wahba (2002) gefordert, keine signifikanten Unterschiede mehr.

Damit fallen alle Tests zum Balancing der Kovariaten positiv aus, so dass die vorgenommenen Matching-Prozeduren als gelungen und die Ergebnisse als gut interpretierbar angesehen werden können.

\subsection{Ergebnisse des Matching-Verfahrens}

Der Ergebnisteil ist in zwei Abschnitte gegliedert. Zunächst wird analysiert, inwieweit Schicht- und Nachtarbeit Gesundheitsindikatoren beeinflusst. Danach wird die Bedeutung der Arbeitsplatzmerkmale für das gesundheitliche Befinden der Beschäftigten untersucht.

\subsubsection{Auswirkungen der Art der Beschäftigung}

Die Ergebnisse zu den Auswirkungen der Art der Beschäftigung sind in Tab. 2 dargestellt. Hier können die Wahrscheinlichkeitsangaben, die auf binär codierten Variablen basieren, als Prozentwerte interpretiert werden. Als wichtiger Befund kann zunächst festgehalten werden, dass bei Kontrolle von soziodemographischen sowie arbeitsplatzbezogenen Merkmalen nur wenige signifikante Zusammenhänge bestehen. Jedoch zeigt sich auch, dass häufige Nachtarbeit die Wahrscheinlichkeit von Schlafstörungen etwa um das 2,3-fache im Vergleich zur Kontrollgruppe steigert. Somit sind ca. $37 \%$ der Beschäftigten mit Nachtarbeit in 5 oder mehr Nächten im Monat von Schlafstörungen betroffen. Um das 1,6-fache und damit höchst signifikant größer ist in der Nachtarbeit von 1 bis 4 Nächten die Wahrscheinlichkeit von Schlafstörungen mit einem Wert von ca. $26 \%$. Aber auch Schichtarbeit erhöht das Risiko von Schlafstörungen signifikant um etwa das 1,3-fache auf knapp $22 \%$. In den jeweiligen Vergleichsgruppen, die direkt vergleichbar belastende Tätigkeiten ausüben und die analoge soziodemographische Merkmale wie die in Schicht- und Nachtarbeit Beschäftigten aufweisen, geben ca. $16 \%$ an, nach der Arbeit häufig unter Schlafstörungen zu leiden. Dies entspricht in etwa dem Durchschnitt der Vollzeiterwerbstätigen der BIBB/BAuA-Erwerbstätigenbefragung. Folglich sind Schlafstörungen eindeutig auf Schichtarbeit, die frühe Morgen- und Abendstunden einschließt und noch einmal mehr auf Nachtarbeit zurückzuführen.

Ein zweiter auffälliger Befund betrifft die signifikante Zunahme von Nervosität oder Reizbarkeit, aber auch von Burnout sowie von kardiovaskulären Beschwerden in der Gruppe der Nachtarbeit zwischen 1 und 4 Nächten. In der Dokumentation der Logit-Modelle wurde darauf hingewiesen, dass diese Gruppe im Vergleich zu den anderen Schicht- und Nachtarbeitsgruppen durchschnittlich etwas älter und zugleich besser qualifiziert ist und in diesen Merkmalen dem Durchschnitt der Vollzeiterwerbstätigen in Nicht-Schichtarbeit entspricht. Gesundheitsberufe sind in dieser Nachtarbeitsgruppe überdurchschnittlich häufig zu finden. Entsprechend werden von dieser Gruppe sowie von ihrer Kontrollgruppe verantwortungsvolle Aufgaben sowie Arbeiten mit Gefahrenstoffen oder unter Leistungsdruck ausgeführt. Es konnte erwartet werden, dass in dieser Gruppe die eher geringe monatliche Häufigkeit von Nachtarbeit gepaart mit geringen körperlichen Belastungsfaktoren jedoch vergleichsweise größeren Handlungsspielräumen und höherer Qualifikation, die wiederum gesundheitsförderliche Kontrollüberzeugungen fördern, mit eher unauffälligen gesundheitlichen Beeinträchtigungen einhergeht. So bestehen in diesen Gruppen auch weitgehend keine überdurchschnittlichen physischen oder sozialen gesundheitlichen Beanspruchungen. Allerdings sind in der Gruppe der Nachtarbeit zwischen 1 und 4 Nächten die psychischen Beanspruchungen 
Tab. 2 Übersicht über die Ergebnisse des Matchings

\begin{tabular}{|c|c|c|c|c|c|c|c|}
\hline & \multicolumn{7}{|c|}{ Schichtarbeit (Tagschichten zwischen 5 bis 23 Uhr) und Vergleichsgruppe } \\
\hline & Beanspruchung & Schichtarbeit & Vergleichsgruppe & Differenz & Std. Err. & $Z$-Wert & Fälle $^{1}$ \\
\hline \multirow[t]{5}{*}{ Schmerzen Skelett } & Rücken & 0,468 & 0,454 & 0,014 & 0,023 & 0,60 & 1012 \\
\hline & Nacken & 0,470 & 0,458 & 0,012 & 0,025 & 0,48 & 1012 \\
\hline & Arme/Hände & 0,246 & 0,230 & 0,016 & 0,019 & 0,86 & 1012 \\
\hline & Hüfte & 0,106 & 0,117 & $-0,012$ & 0,013 & $-0,88$ & 1012 \\
\hline & Knie & 0,212 & 0,201 & 0,012 & 0,018 & 0,64 & 1012 \\
\hline \multirow[t]{4}{*}{ Psyche } & Nervosität/Reizbarkeit & 0,298 & 0,272 & 0,027 & 0,020 & 1,37 & 1012 \\
\hline & Niedergeschlagenheit & 0,223 & 0,213 & 0,010 & 0,017 & 0,62 & 1012 \\
\hline & Burnout & 0,070 & 0,071 & $-0,000$ & 0,011 & $-0,03$ & 1011 \\
\hline & Depressionen & 0,045 & 0,043 & 0,003 & 0,009 & 0,30 & 1011 \\
\hline \multicolumn{2}{|c|}{ Probleme Kardiosystem } & 0,048 & 0,049 & $-0,001$ & 0,009 & $-0,10$ & 1012 \\
\hline \multicolumn{2}{|l|}{ Schlafstörungen } & 0,216 & 0,161 & $0,056^{* *}$ & 0,018 & 3,05 & 1012 \\
\hline \multicolumn{2}{|c|}{ Magen-Darm-Probleme } & 0,119 & 0,106 & 0,013 & 0,014 & 0,94 & 1012 \\
\hline
\end{tabular}

\begin{tabular}{|c|c|c|c|c|c|c|c|}
\hline & \multicolumn{7}{|c|}{ Nachtarbeit in 1 bis 4 Nächten im Monat ( 23 bis 5 Uhr) und Vergleichsgruppe } \\
\hline & Beanspruchung & Nachtschicht 1 bis 4 Nächte & Vergleichsgruppe & Differenz & Std. Err. & Z-Wert & Fälle $^{1}$ \\
\hline \multirow[t]{5}{*}{ Schmerzen Skelett } & Rücken & 0,401 & 0,405 & $-0,004$ & 0,023 & $-0,31$ & 679 \\
\hline & Nacken & 0,434 & 0,464 & $-0,030$ & 0,025 & $-1,17$ & 679 \\
\hline & Arme/Hände & 0,138 & 0,153 & $-0,015$ & 0,018 & $-1,57$ & 679 \\
\hline & Hüfte & 0,093 & 0,087 & 0,006 & 0,017 & 0,34 & 679 \\
\hline & Knie & 0,158 & 0,161 & $-0,003$ & 0,020 & $-0,19$ & 679 \\
\hline \multirow[t]{4}{*}{ Psyche } & Nervosität/Reizbarkeit & 0,321 & 0,273 & $0,048^{*}$ & 0,024 & 2,01 & 679 \\
\hline & Niedergeschlagenheit & 0,202 & 0,198 & 0,003 & 0,020 & 0,17 & 679 \\
\hline & Burnout & 0,126 & 0,075 & $0,050^{* *}$ & 0,016 & 3,11 & 677 \\
\hline & Depressionen & 0,046 & 0,042 & 0,004 & 0,010 & 0,39 & 679 \\
\hline \multicolumn{2}{|c|}{ Probleme Kardiosystem } & 0,065 & 0,041 & $0,023^{*}$ & 0,012 & 2,04 & 679 \\
\hline \multicolumn{2}{|c|}{ Schlafstörungen } & 0,261 & 0,161 & $0,100^{* * *}$ & 0,022 & 4,63 & 679 \\
\hline \multicolumn{2}{|c|}{ Magen-Darm-Probleme } & 0,127 & 0,108 & 0,018 & 0,017 & 1,07 & 679 \\
\hline & \multicolumn{7}{|c|}{ Nachtarbeit in 5 oder mehr Nächten im Monat ( 23 bis $5 \mathrm{Uhr}$ ) und Vergleichsgruppe } \\
\hline & Beanspruchung & Nachtschicht $\geq 5$ Nächte & Vergleichsgruppe & Differenz & Std. Err. & $Z$-Wert & Fälle $^{1}$ \\
\hline \multirow[t]{5}{*}{ Schmerzen Skelett } & Rücken & 0,447 & 0,471 & $-0,024$ & 0,023 & $-1,06$ & 1118 \\
\hline & Nacken & 0,419 & 0,444 & $-0,025$ & 0,020 & $-1,46$ & 1118 \\
\hline & Arme/Hände & 0,237 & 0,220 & 0,017 & 0,020 & 0,81 & 1118 \\
\hline & Hüfte & 0,112 & 0,112 & $-0,001$ & 0,013 & $-0,04$ & 1118 \\
\hline & Knie & 0,226 & 0,207 & 0,020 & 0,018 & 1,10 & 1118 \\
\hline \multirow[t]{4}{*}{ Psyche } & Nervosität/Reizbarkeit & 0,309 & 0,293 & 0,016 & 0,019 & 0,83 & 1116 \\
\hline & Niedergeschlagenheit & 0,233 & 0,212 & 0,021 & 0,016 & 1,30 & 1117 \\
\hline & Burnout & 0,088 & 0,082 & 0,004 & 0,012 & 0,37 & 1118 \\
\hline & Depressionen & 0,047 & 0,045 & 0,002 & 0,010 & 0,20 & 1118 \\
\hline \multicolumn{2}{|c|}{ Probleme Kardiosystem } & 0,051 & 0,048 & 0,003 & 0,009 & 0,29 & 1118 \\
\hline \multicolumn{2}{|c|}{ Schlafstörungen } & 0,374 & 0,162 & $0,213^{* * *}$ & 0,018 & 11,60 & 1117 \\
\hline \multicolumn{2}{|c|}{ Magen-Darm-Probleme } & 0,139 & 0,101 & $0,037^{* *}$ & 0,015 & 2,53 & 1118 \\
\hline
\end{tabular}

${ }^{*} p<0.05,{ }^{* *} p<0.01,{ }^{* * *} p<0.001$

Quelle: BIBB/BAUA-Erwerbstätigenbefragung 2006, alle Angaben gewichtet, eigene Berechnungen 
Tab. 3 Ergebnisse des Matchings im Vergleich zu allen Vollzeitbeschäftigten

\begin{tabular}{|c|c|c|c|c|c|c|c|c|}
\hline & Beanspruchung & $\begin{array}{l}\text { Schicht- } \\
\text { arbeit }\end{array}$ & $\begin{array}{l}\text { Vergleichs- } \\
\text { gruppe }\end{array}$ & $\begin{array}{l}\text { Nachtarbeit } 1 \\
\text { bis } 4 \text { Nächte }\end{array}$ & $\begin{array}{l}\text { Vergleichs- } \\
\text { gruppe }\end{array}$ & $\begin{array}{l}\text { Nachtarbeit } \\
\geq 5 \text { Nächte }\end{array}$ & $\begin{array}{l}\text { Vergleichs- } \\
\text { gruppe }\end{array}$ & $\begin{array}{l}\text { Vollzeit- } \\
\text { beschäftigte }\end{array}$ \\
\hline \multirow[t]{5}{*}{ Schmerzen Skelett } & Rücken & $0,468^{* * *}$ & $0,454^{* * *}$ & 0,401 & 0,405 & $0,447^{* * *}$ & $0,471^{* * *}$ & 0,383 \\
\hline & Nacken & $0,470^{* *}$ & $0,458^{*}$ & 0,434 & 0,464 & 0,419 & 0,444 & 0,442 \\
\hline & Arme/Hände & $0,246^{* * *}$ & $0,230^{* * *}$ & 0,138 & 0,153 & $0,237^{* * *}$ & $0,220^{* *}$ & 0,146 \\
\hline & Hüfte & $0,106^{* * *}$ & $0,117^{* * *}$ & 0,093 & 0,087 & $0,112^{* * *}$ & $0,112^{* * *}$ & 0,075 \\
\hline & Knie & $0,212^{* * *}$ & $0,201^{* * *}$ & 0,158 & 0,161 & $0,226^{* * *}$ & $0,207^{* *}$ & 0,133 \\
\hline \multirow[t]{4}{*}{ Psyche } & Nervosität/Reizbarkeit & $0,298^{*}$ & 0,272 & $0,321^{* * *}$ & 0,273 & $0,309^{* * *}$ & $0,293^{* *}$ & 0,262 \\
\hline & Niedergeschlagenheit & $0,223^{* * *}$ & $0,213^{* * *}$ & $0,202^{* *}$ & $0,198^{* *}$ & $0,233^{* * *}$ & $0,212^{* *}$ & 0,161 \\
\hline & Burnout & 0,070 & 0,071 & $0,126^{* * *}$ & 0,075 & 0,088 & 0,082 & 0,074 \\
\hline & Depressionen & $0,045^{*}$ & $0,043^{*}$ & $0,046^{*}$ & $0,042^{*}$ & $0,047^{* *}$ & $0,045^{*}$ & 0,033 \\
\hline \multicolumn{2}{|c|}{ Probleme Kardiosystem } & 0,048 & 0,049 & $0,065^{*}$ & 0,041 & 0,051 & 0,048 & 0,047 \\
\hline \multicolumn{2}{|c|}{ Schlafstörungen } & $0,216^{* * *}$ & 0,161 & $0,261^{* * *}$ & 0,161 & $0,374^{* * *}$ & 0,162 & 0,163 \\
\hline \multicolumn{2}{|c|}{ Magen-Darm-Probleme } & $0,119^{*}$ & 0,106 & $0,127^{*}$ & 0,108 & $0,139^{* * *}$ & 0,101 & 0,097 \\
\hline
\end{tabular}

${ }^{1}$ Die Signifikanten beruhen auf $t$-Tests für die Unterschiede zwischen der jeweiligen Gruppe und der Gruppe der Vollzeitbeschäftigten. ${ }^{*} p<0.05$, ${ }^{* *} p<0.01,{ }^{* * *} p<0.001$

Quelle: BIBB/BAUA-Erwerbstätigenbefragung 2006, alle Angaben gewichtet, eigene Berechnungen

wie Nervosität und Reizbarkeit signifikant sowie Burnout hoch signifikant abweichend von der Kontrollgruppe. Dies ist ein Hinweis darauf, dass diese genannten spezifischen Arbeitsanforderungen in Verbindung mit der Lage der Arbeitszeit gesundheitliche Beeinträchtigungen hervorrufen.

Ein dritter Befund betrifft die signifikante Zunahme von gastrointestinalen Beschwerden in der Gruppe der häufigen Nachtarbeit. Diese Gruppe ist vergleichsweise häufig im produzierenden Gewerbe und in Verkehrsgewerbe vertreten, geringer qualifiziert und stärker durch physische und soziale Belastungen am Arbeitsplatz gekennzeichnet. Somit deutet sich an, dass auch Magen-Darm-Beschwerden auf die Lage der Arbeitszeit zurückzuführen sind.

Hinsichtlich von Skeletterkrankungen sowie den psychischen Gesundheitsfolgen „Niedergeschlagenheit“ und „Depressionen“ ergeben sich demgegenüber keine signifikanten Unterschiede zwischen den Schicht- und Nachtarbeitsgruppen und ihren vergleichbaren Kontrollgruppen.

Als Zwischenfazit lässt sich somit festhalten: Schicht- und Nachtarbeit in 5 oder mehr Nächten im Monat hat mit Ausnahme von Schlafstörungen sowie von gastrointestinalen Beschwerden in der zuletzt genannten Gruppe keinen eigenständigen Effekt auf selbstwahrgenommene gesundheitliche Beschwerden bei abhängig beschäftigten Vollzeitarbeitnehmern in überdurchschnittlich belastenden Tätigkeiten. Nachtarbeiter in 1 bis 4 Nächten im Monat weisen zusätzlich zu Schlafstörungen auch psychische Beschwerden wie Nervosität, Reizbarkeit und Burnout sowie kardiovaskuläre Probleme auf. Hier besteht offensichtlich ein Zusammenhang zwischen den in dieser Nachtarbeitsgruppe vor- herrschenden spezifischen Arbeitsanforderungen, die neben höheren Handlungsspielräumen, durch verantwortungsvolle Aufgaben, Arbeit mit Gefahrenstoffen und Leistungsdruck gekennzeichnet sind, sowie der Lage der Arbeitszeit und den gesundheitlichen Beeinträchtigungen. Mitgliedern dieser Gruppe gelingt es, ungeachtet ihrer vergleichsweise höheren Qualifikation und durchaus auch entlastenden höheren Autonomie, offenbar nicht die Belastungsmomente ohne gesundheitliche Beanspruchungen zu bewältigen.

\subsubsection{Bedeutung von Arbeitsplatzmerkmalen}

Die gesundheitlichen Gefährdungen insbesondere von Nachtarbeit basieren vor allem auf Drittvariablen und hier vorwiegend auf belastenden Tätigkeitsmerkmalen, die unabhängig von der Lage der Arbeitszeit auch für andere Beschäftigtengruppen negative physische und psychische Gesundheitseffekte hervorrufen. Um dies zu verdeutlichen, werden in Tab. 3 die zuvor dokumentierten Wahrscheinlichkeiten mit denen des Durchschnitts aller in Abschn. 3 definierten Vollzeitbeschäftigten verglichen. So wird deutlich, dass die höher belastenden Tätigkeitsmerkmale und Arbeitsbedingungen, denen Schicht- und Nachtarbeiter vergleichsweise häufiger ausgesetzt sind (siehe Abschn. 4.1) und die in der vorliegenden Analyse zum Abgleich der Kontrollgruppen der Nicht-Schichtarbeit herangezogen wurden, einzelne, die Gesundheit beeinträchtigende Effekte bedingen.

Dies gilt zunächst kaum für kardiovaskuläre Beschwerden, die während oder direkt nach der Arbeit auftreten. Sie werden von allen Gruppen - mit Ausnahme der schon zuvor berichteten höheren Beeinträchtigung bei Nachtarbeit in 1 
bis 4 Nächten im Monat - ähnlich häufig wie vom Durchschnitt der Vollzeitbeschäftigten (4,7 \%) genannt.

Anders ist es zum Teil bei Fragen nach psychischen Beschwerden, die häufig während oder direkt nach der Arbeit auftreten. Hier erwähnen die Befragten sehr häufig Nervosität oder Reizbarkeit. Auf den signifikant höheren Effekt bei den Beschäftigten in Nachtarbeit von 1 bis 4 Nächten gegenüber der strukturgleichen Kontrollgruppe wurde zuvor bereits eingegangen. Doch auch der Wert der Schichtarbeit ist mit etwa $30 \%$ signifikant höher als der des Durchschnitts aller Vollzeiterwerbstätigen von etwa $26 \%$. Insbesondere bei der Nachtarbeitsgruppe in 5 oder mehr Nächten, aber eben auch in der entsprechenden Kontrollgruppe, sind hoch und höchst signifikante Abweichungen feststellbar.

Beachtenswert sind zudem drei weitere Effekte: Erstens geben Beschäftigte in Schicht- und in Nachtarbeit sowie Erwerbstätige in den strukturgleichen Kontrollgruppen mindestens hoch signifikant häufiger an niedergeschlagen zu sein. Analog dazu treten signifikant öfters Probleme in Bezug auf Depressionen im Vergleich zu allen Vollzeitbeschäftigten auf. Zweitens besteht ein auffälliger Effekt bei Burnout. Dieser wurde entsprechend der Interviewanweisung der Befragung definiert ,als ein chronischer Erschöpfungszustand mit Krankheitsgefühl, der bereits seit sechs Monate andauert" (BIBB/BAuA 2006). Bei Schichtarbeit und bei häufiger Nachtarbeit und ihren jeweiligen Kontrollgruppen bestehen keine signifikanten Abweichungen zum Durchschnittswert von 7,4 \% bei der Gesamtgruppe der Vollzeitbeschäftigten. Signifikante Burnout-Probleme berichten hingegen Beschäftigte in Nachtarbeit von 1 bis 4 Nächten $(12,6 \%)$, während die strukturgleiche Kontrollgruppe mit 7,5\% keine signifikante Abweichung aufweist. Beide Gruppen sind jedoch, wie zuvor beschrieben, vor allem durch vergleichsweise hohe psychische Belastungen gekennzeichnet. Dies stützt die bereits getroffene Annahme, dass die Lage der Arbeitszeit in Verbindung mit diesen spezifischen Belastungen die Wahrscheinlichkeit von Burnout-Symptomen in dieser Gruppe spürbar fördert.

Vergleichbar verhält es sich bei gastrointestinalen Beschwerden. In der Gruppe der Schichtarbeit berichten knapp $12 \%$ von Magen-Darm-Problemen während oder unmittelbar nach der Arbeit. Dieser Wert liegt damit signifikant höher als im Durchschnitt von 9,7 \% der Vollzeitbeschäftigten. Bei den Beschäftigten in Nachtarbeit bis zu 4 Nächten im Monat sind es 12,7 \%, die ungeachtet der guten Behandlungsmöglichkeiten, häufige gastrointestinale Beschwerden mitteilen. In der Gruppe der Personen mit häufiger Nachtarbeit liegt der Anteil mit 13,9\% allerdings höchst signifikant über dem Durchschnitt aller Vollzeitbeschäftigten. Dies korrespondiert mit den ebenfalls in den Schicht- und Nachtarbeitsgruppen höchst signifikant häufiger genannten Schlafstörungen. Demzufolge sind die Schlafstörungen sowie damit einhergehende Magen-Darm-Probleme auf die Lage der Arbeitszeit zurückzuführen.
Insbesondere physische Belastungen bewirken einzelne Skeletterkrankungen. In den Gruppen der in Schichtarbeit und in häufiger Nachtschichtarbeit Tätigen sowie ihren nicht in Schicht- bzw. Nachtschicht arbeitenden Vergleichsgruppen bestehen sehr deutliche Beanspruchungen in Form von Schmerzen im Rücken (über $45 \%$ ), in Armen oder Händen (über $22 \%$ ), in Knien (über $20 \%$ ) oder in der Hüfte (über $11 \%$ ). Die Unterschiede dieser Gruppen sind hoch bzw. höchst signifikant verschieden vom Durchschnitt der Vollzeitbeschäftigten. Da ausschließlich Beschäftigte in Schicht- und häufiger Nachtarbeit bzw. ihre Kontrollgruppen von Schmerzen berichten, sind es vor allem physisch und sozial hoch belastende Arbeitsplatz- und Tätigkeitsmerkmale der zumeist produktiv oder in Verkehrsberufen Beschäftigten, welche diese Rücken- und Gelenkbeschwerden erklären.

\section{Resümee}

In vielen Untersuchungen werden gesundheitliche Gefährdungen durch Schicht- und Nachtarbeit berichtet. Insbesondere Metastudien kritisieren jedoch immer wieder die unzureichende Kontrolle von soziodemographischen Einflussfaktoren sowie von Arbeitsplatz- und Tätigkeitsmerkmalen.

Anhand der BIBB/BAuA-Erwerbstätigenbefragung 2006 wurde die Wirkung von zahlreichen tätigkeitsspezifischen und sozialstrukturellen Einflussfaktoren auf das physische und psychische Befinden sowie auf Schlafstörungen untersucht. Die Analyse erfolgte mit einem Matching-Ansatz. Hierbei wurden zu Schicht- und Nachtarbeitsgruppen in Bezug auf sozialstrukturelle Charakteristika und Tätigkeitsmerkmale möglichst strukturgleiche Kontrollgruppen gebildet, um eigenständige Effekte der Lage der Arbeitszeit auf gesundheitliche Beeinträchtigungen zu extrahieren.

Insgesamt zeigen die Ergebnisse, dass Schicht- und insbesondere Nachtarbeit die Wahrscheinlichkeit von Schlafstörungen deutlich erhöht. Häufige Nachtarbeit, die vor allem durch physische und soziale Arbeitsbelastungen gekennzeichnet ist, scheint einen Effekt auf Magen-DarmBeschwerden auszuüben. Seltenere Nachtarbeit, die durch anspruchsvolle Arbeitsanforderungen wie verantwortungsvolle Aufgaben, Arbeit mit Gefahrenstoffen und Leistungsdruck gekennzeichnet ist, wirkt zudem negativ auf psychische Arbeitsbelastungen in Form von Nervosität und Reizbarkeit oder Burnout sowie auf karidovaskuläre Probleme. Für weitere gesundheitliche Effekte und hier insbesondere für Schmerzen im Skelettbereich, die (exklusiv) durch die Lage der Arbeitszeit oder indirekt über Schlafstörungen und Schlafmangel bei Schicht- und Nachtarbeit verursacht werden, bestehen keine Hinweise.

Neben diesen Beeinträchtigungen der Gesundheit können allerdings noch dauerhafte gesundheitliche Folgen ins- 
besondere aufgrund von verminderten Regenerationsmöglichkeiten bei Nachtarbeit erwartet werden. Dies gilt umso mehr, als Schicht- und Nachtarbeit in der Regel mit weiteren Belastungen am Arbeitsplatz einhergehen, die sich dann in physischen Beschwerden oder auch Niedergeschlagenheit und Depressionen ausdrücken.

Sehr deutlich offenbaren sich allerdings psychische und physische Gesundheitsbeeinträchtigungen durch Tätigkeitsmerkmale und Arbeitsbedingungen. Dies zeigen die Ergebnisse zu Gesundheitsbeeinträchtigungen, die sowohl Personen in Schicht- und Nachtarbeit als auch jene Kontrollpersonen betreffen, welche in vergleichbaren Tätigkeiten tagsüber arbeiten. Anhand der hier analysierten Querschnittdaten, die allerdings langfristige Gesundheitseffekte unbeobachtet lassen, kann zugespitzt festgestellt werden: Wichtig für die Gesundheit ist insbesondere die Frage, ob an belastenden Arbeitsplätzen gearbeitet wird. Angesichts dessen ist es etwas weniger wichtig, zu welcher Tages- oder Nachtzeit dies geschieht.

Es bestehen in Deutschland u. a. im Arbeitszeitgesetz gesetzliche Schutzregelungen hinsichtlich der Arbeitszeit von Beschäftigten. Speziell für Nacht- und Schichtarbeitnehmer sind diese ,nach gesicherten arbeitswissenschaftlichen Erkenntnissen über die menschengerechte Gestaltung der Arbeit festzulegen“ (ArbzG § 6, Abs. 2). Zudem sind Ansprüche auf arbeitsmedizinische Untersuchungen und Umsetzungsrechte auf Tagarbeitsplätze festgelegt (ArbzG § 6, Abs. 3 und 4). ${ }^{15}$ Auf Basis von vorliegenden Erkenntnissen der zahlreichen, oben zitierten Teilstudien zu Gesundheitseffekten sowie zu Zeitplänen und Produktivität (Åkerstedt 2003; Folkard und Tucker 2003) wurden darüber hinaus auf nationaler und internationaler Ebene viele Gestaltungsempfehlungen für Schichtarbeit entwickelt. So sollte etwa die Anzahl hintereinander liegender gleicher Schichten auf drei begrenzt sein und die letzte Nachtschicht in zwei freie Tage münden. Bei Rotationen ist auf Vorwärts- wechsel zu achten. Lange Schichtzeiten über 8 Stunden sollten ebenso vermieden werden wie ein sehr früher Schichtbeginn und ein sehr spätes Schichtende. Auch werden Ruhezeiten von 11 Stunden empfohlen usw. (Beermann 2010; Knauth 1993; Wedderburn 2000). Derartige Gestaltungshinweise sind der gesundheitlichen Vorsorge dienlich, zumal sie nicht allein auf Verhaltensänderungen von Beschäftigten vertrauen (Richter et al. 2010), sondern Struktureffekte ernst nehmen.

Angesichts des Ergebnisses, dass belastende Tätigkeiten sehr deutlich - auch unabhängig von der Lage der Arbeitszeit - die Wahrscheinlichkeit für gesundheitliche Beanspruchungen erhöhen, sollte der Blick in stärkerem MaBe jedoch auch auf weitere belastende Tätigkeitsmerkmale gerichtet werden. Dies gilt einerseits für die strukturelle Arbeitsgestaltung in Unternehmen sowie anderseits für die bislang vernachlässigte systematische Berücksichtigung von sozialen, physischen und psychischen Arbeitsplatzbelastungen in wissenschaftlichen Studien zum Gesundheitszustand. Charakteristika des Arbeitsplatzes sowie Tätigkeitsmerkmale beeinflussen die Gesundheit in erheblichem Maße. Gerade diese Faktoren können jedoch von Arbeitgeberseite vergleichsweise gut in positiver Weise beeinflusst werden. Damit würden gesundheitsfördernde Effekte zugleich für Tagarbeit wie auch für Nacht- und Schichtarbeit erzielt. Zudem besteht ein Bedarf an längsschnittlich angelegten Befragungen auf Individualebene, anhand derer kausale und langfristig wirkende Zusammenhänge zwischen Arbeitsplatzmerkmalen, soziodemographischen, familiär-sozialen und individuellen Faktoren, wie etwa persönlichkeitsdifferente Fähigkeiten zur Anpassung an Schicht- oder Nachtarbeit, umfänglicher und systematischer als bislang möglich untersucht werden können. Um effektive Gestaltungsempfehlungen geben zu können, die auf Evidenz basieren, sind vor allem bessere Datengrundlagen aber auch noch viel Forschung erforderlich.

\footnotetext{
${ }^{15}$ Ausnahmeregelungen bieten allerdings erhebliche arbeitszeitgesetzliche Potenziale für Arbeitszeitflexibilisierung (Schlottfeldt 2008: 474).
} 
Anhang: Tabellen 4 bis 9 und Abb. 1

Tab. 4 Logit-Modell - Schichtarbeit

\begin{tabular}{|c|c|c|c|}
\hline \multicolumn{2}{|c|}{ Beschäftigungsart $(0=$ Nicht-Schichtarbeit, $1=$ Schichtarbeit $)$} & \multirow{2}{*}{$\frac{\text { Koeffizient }}{0,460^{* *}}$} & \multirow{2}{*}{$\frac{\text { Std. Err }}{(0,272)}$} \\
\hline Höchster Berufsabschluss & Keine Ausbildung & & \\
\hline (Ref. Ausbildung) $(1=$ ja) & Fachschule & $-0,446^{*}$ & $(0,125)$ \\
\hline & (Fach-)Hochschule & $-0,803^{* * *}$ & $(0,065)$ \\
\hline \multirow{2}{*}{$\begin{array}{l}\text { Altersgruppen } \\
\text { (Ref.: Alter 18-35) }\end{array}$} & Alter $36-50$ & $-0,197$ & $(0,083)$ \\
\hline & Alter $51-65$ & $-0,641^{* * *}$ & $(0,072)$ \\
\hline Geschlecht $(1=$ weiblich $)$ & Geschlecht & $0,279^{* *}$ & $(0,138)$ \\
\hline Familienstand ( 1 = verheiratet $)$ & verheiratet & 0,047 & $(0,108)$ \\
\hline Kinder im HH $(1=$ ja $)$ & Kinder & $-0,093$ & $(0,098)$ \\
\hline Staatsangehörigkeit (1 = Ausl.) & Staatsangehörigkeit & 0,086 & $(0,223)$ \\
\hline \multirow[t]{18}{*}{ Tätigkeitsmerkmale (1= ja) } & Verantwortungsvolle Tätigkeit & 0,066 & $(0,105)$ \\
\hline & Häufiges Arbeiten unter Leistungsdruck & 0,025 & $(0,100)$ \\
\hline & Wenig Handlungsspielraum vorhanden & $0,569^{* * *}$ & $(0,189)$ \\
\hline & Auftreten von Monotonie & $0,256^{* *}$ & $(0,125)$ \\
\hline & Häufig neue Aufgaben & $-0,306$ & $(0,129)$ \\
\hline & Fachliche Überforderung & 0,382 & $(0,341)$ \\
\hline & Fachliche Unterforderung & 0,177 & $(0,160)$ \\
\hline & Überforderung durch Arbeitspensum & $-0,247$ & $(0,101)$ \\
\hline & Unterforderung durch Arbeitspensum & $-0,319$ & $(0,161)$ \\
\hline & Häufiges Arbeiten im Stehen & $0,726^{* * *}$ & $(0,279)$ \\
\hline & Häufiges Arbeiten im Sitzen & $-0,691^{* * *}$ & $(0,061)$ \\
\hline & Häufiges Tragen der Lasten & 0,027 & $(0,114)$ \\
\hline & Häufiges Arbeiten in gebückter Haltung & $-0,093$ & $(0,124)$ \\
\hline & Häufiges Arbeiten mit Erschütterungen & 0,173 & $(0,291)$ \\
\hline & Häufiges Arbeiten unter Belastung der Atemwege & 0,129 & $(0,131)$ \\
\hline & Häufiges Arbeiten mit Gefahrenstoffen & 0,022 & $(0,179)$ \\
\hline & Häufiges Arbeiten bei Lärm & $0,328^{* *}$ & $(0,155)$ \\
\hline & Häufiges Arbeiten mit Mikroorganismen & 0,311 & $(0,236)$ \\
\hline \multirow{6}{*}{$\begin{array}{l}\text { Branchen } \\
\text { (Ref: Prod. Gewerbe) }(1=\mathrm{ja})\end{array}$} & Dienstleistungen (ohne öffentliche) & $-0,138$ & $(0,092)$ \\
\hline & Baugewerbe & $-2,404 * * *$ & $(0,029)$ \\
\hline & Gesundheitswesen & $0,451^{* *}$ & $(0,251)$ \\
\hline & Land- u, Forstwirtschaft & $-1,235^{*}$ & $(0,150)$ \\
\hline & Verkehr & 0,536 & $(0,562)$ \\
\hline & Öffentliche Dienstleistung & $-0,945^{* * *}$ & $(0,108)$ \\
\hline \multicolumn{2}{|l|}{ Konstante } & $-1,875^{* * *}$ & $(0,188)$ \\
\hline \multicolumn{2}{|l|}{ Anzahl der Beobachtungen } & & \\
\hline \multicolumn{2}{|l|}{ Pseudo $R^{2}$} & & \\
\hline \multicolumn{2}{|l|}{ Wald-Test (Freiheitsgrade) } & & \\
\hline \multicolumn{2}{|l|}{ Log-Pseudolikelihood } & & \\
\hline
\end{tabular}

${ }^{*} p<0.05,{ }^{* *} p<0.01,{ }^{* * *} p<0.001$; Cluster-robuste Standardfehler in Klammern

Quelle: BIBB/BAUA-Erwerbstätigenbefragung 2006, alle Angaben gewichtet, eigene Berechnungen 
Tab. 5 Logit-Modell - Nachtarbeit in 1 bis 4 Nächten im Monat

\begin{tabular}{|c|c|c|c|}
\hline \multicolumn{2}{|c|}{ Beschäftigungsart $(0=$ Nicht-Schichtarbeit, $1=$ Nachtarbeit, $1-4$ Nächte $)$} & Koeffizient & Std. Err \\
\hline \multirow{3}{*}{$\begin{array}{l}\text { Höchster Berufsabschluss } \\
\text { (Ref. Ausbildung) }(1=\text { ja) }\end{array}$} & Keine Ausbildung & 0,049 & $(0,262)$ \\
\hline & Fachschule & 0,055 & $(0,231)$ \\
\hline & (Fach-)Hochschule & 0,144 & $(0,151)$ \\
\hline \multirow{2}{*}{$\begin{array}{l}\text { Altersgruppen } \\
\text { (Ref.: Alter 18-35) }\end{array}$} & Alter $36-50$ & $-0,104$ & $(0,108)$ \\
\hline & Alter $51-65$ & $-0,254$ & $(0,134)$ \\
\hline Geschlecht $(1=$ weiblich $)$ & Geschlecht & $-0,689^{* * *}$ & $(0,064)$ \\
\hline Familienstand $(1=$ verheiratet $)$ & verheiratet & $-0,112$ & $(0,120)$ \\
\hline Kinder im HH (1 = ja) & Kinder & 0,050 & $(0,139)$ \\
\hline Staatsangehörigkeit (1 = Ausl.) & Staatsangehörigkeit & 0,112 & $(0,306)$ \\
\hline \multirow[t]{18}{*}{ Tätigkeitsmerkmale $(1=$ ja $)$} & Verantwortungsvolle Tätigkeit & $0,737^{* * *}$ & $(0,292)$ \\
\hline & Häufiges Arbeiten unter Leistungsdruck & 0,204 & $(0,147)$ \\
\hline & Wenig Handlungsspielraum vorhanden & 0,194 & $(0,162)$ \\
\hline & Auftreten von Monotonie & $-0,139$ & $(0,096)$ \\
\hline & Häufig neue Aufgaben & 0,160 & $(0,201)$ \\
\hline & Fachliche Überforderung & $-0,280$ & $(0,249)$ \\
\hline & Fachliche Unterforderung & 0,135 & $(0,213)$ \\
\hline & Überforderung durch Arbeitspensum & 0,175 & $(0,166)$ \\
\hline & Unterforderung durch Arbeitspensum & $-0,044$ & $(0,288)$ \\
\hline & Häufiges Arbeiten im Stehen & $0,583^{* * *}$ & $(0,269)$ \\
\hline & Häufiges Arbeiten im Sitzen & 0,012 & $(0,142)$ \\
\hline & Häufiges Tragen der Lasten & 0,222 & $(0,201)$ \\
\hline & Häufiges Arbeiten in gebückter Haltung & 0,102 & $(0,214)$ \\
\hline & Häufiges Arbeiten mit Erschütterungen & 0,271 & $(0,371)$ \\
\hline & Häufiges Arbeiten unter Belastung der Atemwege & $-0,069$ & $(0,154)$ \\
\hline & Häufiges Arbeiten mit Gefahrenstoffen & $0,563^{* *}$ & $(0,305)$ \\
\hline & Häufiges Arbeiten bei Lärm & $0,299^{*}$ & $(0,198)$ \\
\hline & Häufiges Arbeiten mit Mikroorganismen & $0,729^{* * *}$ & $(0,362)$ \\
\hline Branchen & Dienstleistungen (ohne öffentliche) & 0,085 & $(0,157)$ \\
\hline (Ref.: Prod. Gewerbe) $(1=$ ja) & Baugewerbe & $-0,978^{* * *}$ & $(0,096)$ \\
\hline & Gesundheitswesen & $1,366^{* * *}$ & $(0,709)$ \\
\hline & Land- u. Forstwirtschaft & $-0,384$ & $(0,416)$ \\
\hline & Verkehr & $1,906^{* * *}$ & $(2,047)$ \\
\hline & Öffentliche Dienstleistung & $0,722^{* * *}$ & $(0,398)$ \\
\hline Konstante & & $-3,535^{* * *}$ & $(0,232)$ \\
\hline Anzahl der Beobachtungen & $71^{\prime}$ & & \\
\hline Pseudo $R^{2}$ & 0,1 & & \\
\hline Wald-Test (Freiheitsgrade) & 387,15 & & \\
\hline Log-Pseudolikelihood & -193 & & \\
\hline
\end{tabular}

${ }^{*} p<0.05,{ }^{* *} p<0.01,{ }^{* * *} p<0.001$; Cluster-robuste Standardfehler in Klammern

Quelle: BIBB/BAUA-Erwerbstätigenbefragung 2006, alle Angaben gewichtet, eigene Berechnungen 
Tab. 6 Logit-Modell - Nachtarbeit in 5 oder mehr Nächten im Monat

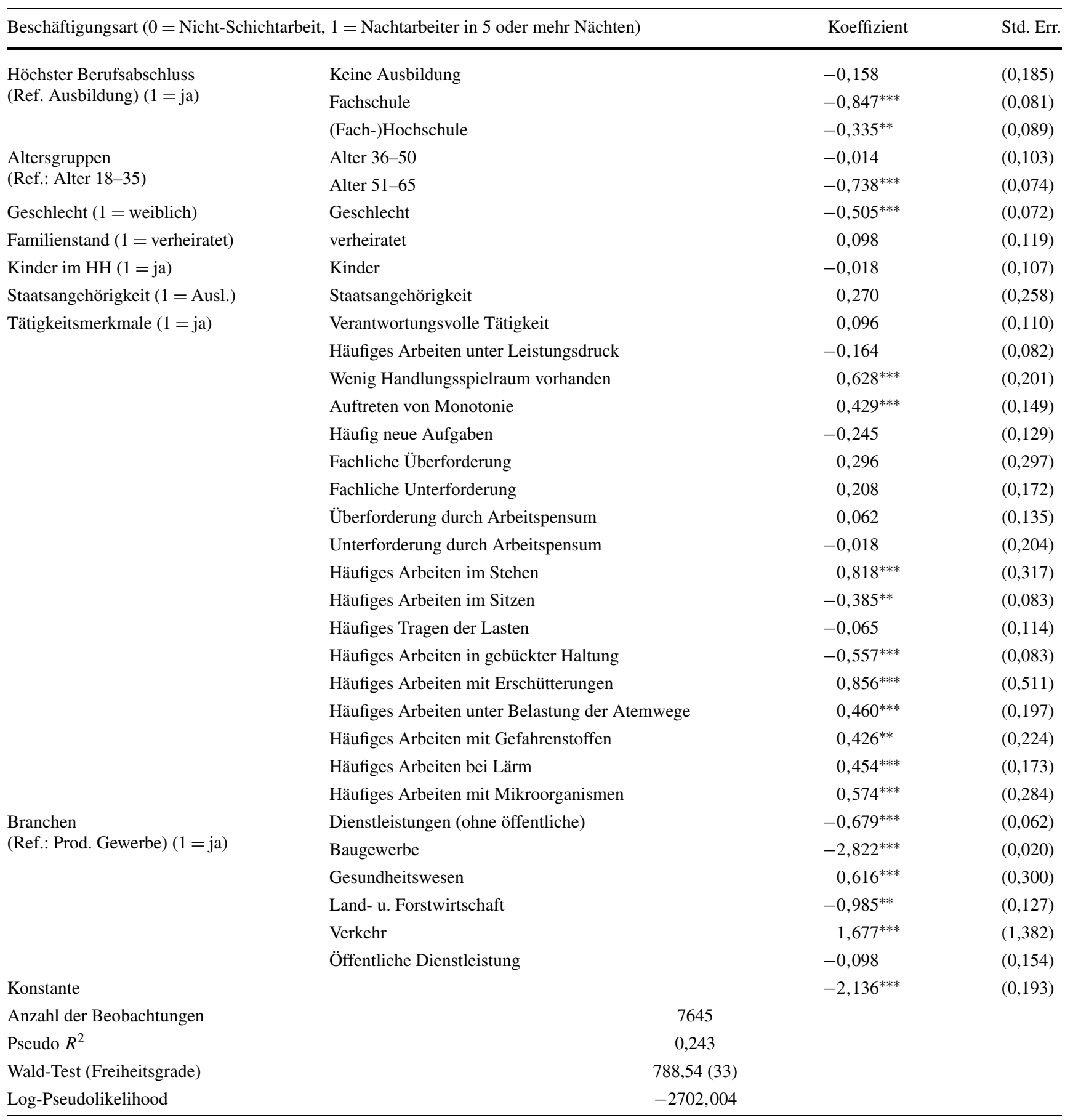

${ }^{*} p<0.05,{ }^{* *} p<0.01,{ }^{* * *} p<0.001$; Cluster-robuste Standardfehler in Klammern

Quelle: BIBB/BAUA-Erwerbstätigenbefragung 2006, alle Angaben gewichtet, eigene Berechnungen 

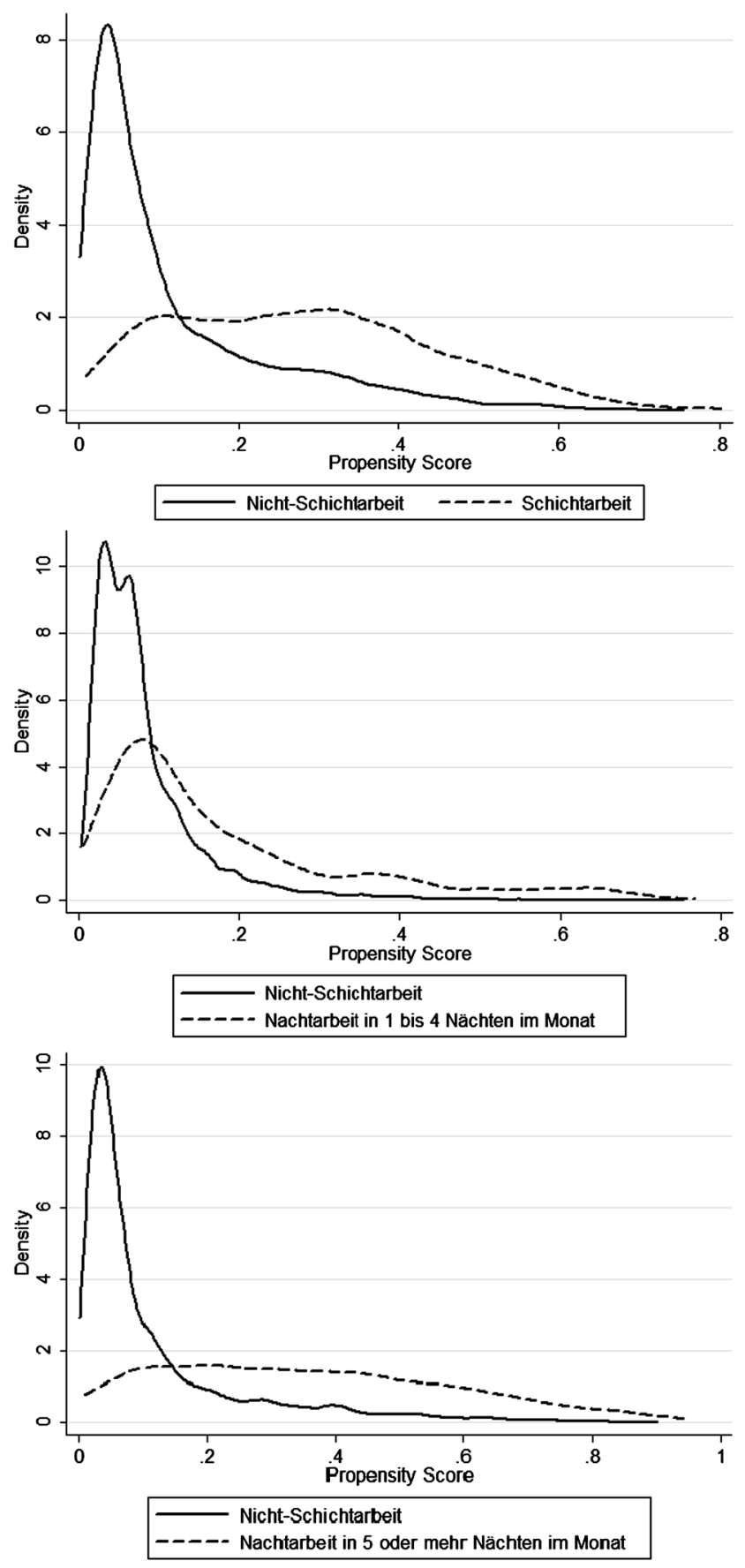

Abb. 1 Kern-Dichte-Schätzer der Verteilungen der Propensity Scores in den jeweiligen Beschäftigtengruppen vor dem Matching (Quelle: BIBB/BAUA-Erwerbstätigenbefragung 2006, eigene Berechnungen) 
Tab. 7 Deskriptive Statistiken und Balancing der Variablen - Schichtarbeit und Vergleichsgruppe

\begin{tabular}{|c|c|c|c|c|c|c|}
\hline \multirow[t]{2}{*}{ Merkmal } & \multirow[t]{2}{*}{ Sample } & \multicolumn{2}{|l|}{ Mittelwert } & \multirow[t]{2}{*}{ SB } & \multirow[t]{2}{*}{$t$-Wert } & \multirow[t]{2}{*}{$p>|t|$} \\
\hline & & Schichtarbeit & Nicht-Schichtarbeit & & & \\
\hline \multirow[t]{2}{*}{ Keine Ausbildung } & Vor Matching & 0,098 & 0,049 & 19,1 & 6,54 & 0,000 \\
\hline & Nach Matching & 0,092 & 0,096 & $-1,4$ & $-0,28$ & 0,776 \\
\hline \multirow[t]{2}{*}{ Ausbildung } & Vor Matching & 0,763 & 0,550 & 46,1 & 13,12 & 0,000 \\
\hline & Nach Matching & 0,765 & 0,763 & 0,5 & 0,11 & 0,910 \\
\hline \multirow[t]{2}{*}{ Fachschule } & Vor Matching & 0,052 & 0,083 & $-12,4$ & $-3,47$ & 0,001 \\
\hline & Nach Matching & 0,053 & 0,059 & $-2,4$ & $-0,58$ & 0,564 \\
\hline \multirow[t]{2}{*}{ (Fach-)Hochschulabschluss } & Vor Matching & 0,087 & 0,319 & $-60,3$ & $-15,66$ & 0,000 \\
\hline & Nach Matching & 0,090 & 0,082 & 1,9 & 0,60 & 0,550 \\
\hline \multirow[t]{2}{*}{ Alter $18-35$} & Vor Matching & 0,400 & 0,317 & 17,3 & 5,30 & 0,000 \\
\hline & Nach Matching & 0,398 & 0,417 & $-3,9$ & $-0,85$ & 0,394 \\
\hline \multirow[t]{2}{*}{ Alter $36-50$} & Vor Matching & 0,451 & 0,483 & $-6,4$ & $-1,91$ & 0,056 \\
\hline & Nach Matching & 0,450 & 0,450 & 0,0 & 0,00 & 0,999 \\
\hline \multirow[t]{2}{*}{ Alter 51-65 } & Vor Matching & 0,149 & 0,200 & $-13,5$ & $-3,89$ & 0,000 \\
\hline & Nach Matching & 0,152 & 0,134 & 4,9 & 1,20 & 0,232 \\
\hline \multirow[t]{2}{*}{ Geschlecht } & Vor Matching & 0,459 & 0,397 & 12,6 & 3,82 & 0,000 \\
\hline & Nach Matching & 0,451 & 0,456 & $-1,2$ & $-0,26$ & 0,793 \\
\hline \multirow[t]{2}{*}{ Verheiratet } & Vor Matching & 0,440 & 0,489 & $-9,8$ & $-2,95$ & 0,003 \\
\hline & Nach Matching & 0,447 & 0,429 & 3,6 & 0,82 & 0,415 \\
\hline \multirow[t]{2}{*}{ Kinder im $\mathrm{HH}$} & Vor Matching & 0,366 & 0,371 & $-1,1$ & $-0,34$ & 0,737 \\
\hline & Nach Matching & 0,364 & 0,342 & 4,6 & 1,05 & 0,295 \\
\hline \multirow[t]{2}{*}{ Staatsangehörigkeit Deutsch } & Vor Matching & 0,046 & 0,029 & 8,8 & 2,86 & 0,004 \\
\hline & Nach Matching & 0,046 & 0,043 & 1,7 & 0,35 & 0,727 \\
\hline \multirow[t]{2}{*}{ Verantwortungsvolle Tätigkeit } & Vor Matching & 0,586 & 0,671 & $-17,6$ & $-5,37$ & 0,000 \\
\hline & Nach Matching & 0,587 & 0,600 & $-2,7$ & $-0,59$ & 0,555 \\
\hline \multirow[t]{2}{*}{ Häufiges Arbeiten unter Leistungsdruck } & Vor Matching & 0,564 & 0,589 & $-4,9$ & $-1,48$ & 0,138 \\
\hline & Nach Matching & 0,562 & 0,547 & 3,1 & 0,69 & 0,491 \\
\hline \multirow[t]{2}{*}{ Wenig Handlungsspielraum vorhanden } & Vor Matching & 0,331 & 0,170 & 37,9 & 12,41 & 0,000 \\
\hline & Nach Matching & 0,315 & 0,308 & 1,8 & 0,37 & 0,711 \\
\hline \multirow[t]{2}{*}{ Auftreten von Monotonie } & Vor Matching & 0,625 & 0,418 & 42,2 & 12,59 & 0,000 \\
\hline & Nach Matching & 0,617 & 0,604 & 2,7 & 0,60 & 0,548 \\
\hline \multirow[t]{2}{*}{ Häufige neue Aufgaben } & Vor Matching & 0,083 & 0,089 & $-2,1$ & $-0,62$ & 0,536 \\
\hline & Nach Matching & 0,083 & 0,082 & 0,4 & 0,08 & 0,935 \\
\hline \multirow[t]{2}{*}{ Fachliche Überforderung } & Vor Matching & 0,050 & 0,035 & 7,2 & 2,29 & 0,022 \\
\hline & Nach Matching & 0,046 & 0,048 & $-0,8$ & $-0,17$ & 0,863 \\
\hline Fachliche Unterforderung & Vor Matching & 0,171 & 0,112 & 17,0 & 5,48 & 0,000 \\
\hline & Nach Matching & 0,169 & 0,158 & 3,1 & 0,66 & 0,511 \\
\hline Überforderung durch Arbeitspensum & Vor Matching & 0,178 & 0,178 & $-0,2$ & $-0,05$ & 0,956 \\
\hline & Nach Matching & 0,174 & 0,166 & 2,1 & 0,48 & 0,635 \\
\hline Unterforderung durch Arbeitspensum & Vor Matching & 0,054 & 0,045 & 4,3 & 1,33 & 0,182 \\
\hline & Nach Matching & 0,055 & 0,050 & 2,2 & 0,49 & 0,623 \\
\hline Häufiges Arbeiten im Stehen & Vor Matching & 0,773 & 0,386 & 85,1 & 24,29 & 0,000 \\
\hline & Nach Matching & 0,766 & 0,737 & 6,3 & 1,50 & 0,134 \\
\hline Häufiges Arbeiten im Sitzen & Vor Matching & 0,343 & 0,693 & $-74,8$ & $-22,68$ & 0,000 \\
\hline & Nach Matching & 0,353 & 0,364 & $-2,3$ & $-0,51$ & 0,614 \\
\hline
\end{tabular}


Tab. 7 (Fortsetzung)

\begin{tabular}{|c|c|c|c|c|c|c|}
\hline \multirow[t]{2}{*}{ Merkmal } & \multirow[t]{2}{*}{ Sample } & \multicolumn{2}{|l|}{ Mittelwert } & \multirow[t]{2}{*}{ SB } & \multirow[t]{2}{*}{$t$-Wert } & \multirow[t]{2}{*}{$p>\mid t$} \\
\hline & & Schichtarbeit & Nicht-Schichtarbeit & & & \\
\hline \multirow[t]{2}{*}{ Häufiges Tragen der Lasten } & Vor Matching & 0,323 & 0,162 & 38,2 & 12,58 & 0,000 \\
\hline & Nach Matching & 0,317 & 0,297 & 4,8 & 0,99 & 0,322 \\
\hline \multirow[t]{2}{*}{ Häufiges Arbeiten in gebückter Haltung } & Vor Matching & 0,186 & 0,103 & 23,8 & 7,88 & 0,000 \\
\hline & Nach Matching & 0,183 & 0,156 & 7,7 & 1,62 & 0,105 \\
\hline \multirow[t]{2}{*}{ Häufiges Arbeiten mit Erschütterungen } & Vor Matching & 0,050 & 0,030 & 10,4 & 3,43 & 0,001 \\
\hline & Nach Matching & 0,050 & 0,042 & 4,5 & 0,95 & 0,341 \\
\hline \multirow[t]{2}{*}{ Häufiges Arbeiten unter Belastung der Atemwege } & Vor Matching & 0,452 & 0,244 & 44,8 & 14,23 & 0,000 \\
\hline & Nach Matching & 0,450 & 0,434 & 3,4 & 0,72 & 0,470 \\
\hline \multirow[t]{2}{*}{ Häufiges Arbeiten mit Gefahrenstoffe } & Vor Matching & 0,088 & 0,054 & 13,1 & 4,30 & 0,000 \\
\hline & Nach Matching & 0,085 & 0,095 & $-3,9$ & $-0,78$ & 0,436 \\
\hline \multirow[t]{2}{*}{ Häufiges Arbeiten bei Lärm } & Vor Matching & 0,335 & 0,181 & 35,9 & 11,66 & 0,000 \\
\hline & Nach Matching & 0,332 & 0,331 & 0,1 & 0,03 & 0,980 \\
\hline \multirow[t]{2}{*}{ Häufiges Arbeiten mit Mikroorganismen } & Vor Matching & 0,109 & 0,042 & 25,6 & 9,18 & 0,000 \\
\hline & Nach Matching & 0,096 & 0,103 & $-2,7$ & $-0,53$ & 0,598 \\
\hline \multirow[t]{2}{*}{ Dienstleistung (ohne öffentliche) } & Vor Matching & 0,375 & 0,400 & $-5,0$ & $-1,51$ & 0,131 \\
\hline & Nach Matching & 0,385 & 0,363 & 4,5 & 1,03 & 0,303 \\
\hline \multirow[t]{2}{*}{ Baugewerbe } & Vor Matching & 0,017 & 0,081 & $-29,9$ & $-7,45$ & 0,000 \\
\hline & Nach Matching & 0,018 & 0,016 & 0,9 & 0,35 & 0,730 \\
\hline \multirow[t]{2}{*}{ Gesundheitswesen } & Vor Matching & 0,163 & 0,072 & 28,6 & 9,92 & 0,000 \\
\hline & Nach Matching & 0,149 & 0,165 & $-4,9$ & $-0,96$ & 0,336 \\
\hline \multirow[t]{2}{*}{ Land und Forstwirtschaft } & Vor Matching & 0,007 & 0,017 & $-9,9$ & $-2,58$ & 0,010 \\
\hline & Nach Matching & 0,007 & 0,011 & $-3,8$ & $-0,99$ & 0,323 \\
\hline \multirow[t]{2}{*}{ Produzierendes Gewerbe } & Vor Matching & 0,392 & 0,300 & 19,3 & 5,93 & 0,000 \\
\hline & Nach Matching & 0,394 & 0,396 & $-0,4$ & $-0,08$ & 0,932 \\
\hline \multirow[t]{2}{*}{ Verkehr } & Vor Matching & 0,019 & 0,013 & 4,5 & 1,44 & 0,151 \\
\hline & Nach Matching & 0,019 & 0,023 & $-3,0$ & $-0,59$ & 0,553 \\
\hline \multirow[t]{2}{*}{ Öffentliche Dienstleistung } & Vor Matching & 0,027 & 0,116 & $-35,1$ & $-8,83$ & 0,000 \\
\hline & Nach Matching & 0,028 & 0,026 & 0,5 & 0,16 & 0,870 \\
\hline \multirow[t]{2}{*}{ Mittlere standardisierte Verzerrung } & Vor Matching & & 22,9 & & & \\
\hline & Nach Matching & & 2,7 & & & \\
\hline
\end{tabular}

Quelle: BIBB/BAUA-Erwerbstätigenbefragung 2006, alle Angaben gewichtet, eigene Berechnungen 
Tab. 8 Deskriptive Statistiken und Balancing der Variablen - Nachtschicht 1 bis 4 Nächte und Vergleichsgruppe

\begin{tabular}{|c|c|c|c|c|c|c|}
\hline \multirow[t]{2}{*}{ Merkmal } & \multirow[t]{2}{*}{ Sample } & \multicolumn{2}{|l|}{ Mittelwert } & \multirow[t]{2}{*}{ SB } & \multirow[t]{2}{*}{$t$-Wert } & \multirow[t]{2}{*}{$p>\mid t$} \\
\hline & & $\begin{array}{l}\text { Nachtschicht } \\
1 \text { bis } 4 \text { Nächte }\end{array}$ & Nicht-Schichtarbeit & & & \\
\hline \multirow[t]{2}{*}{ Keine Ausbildung } & Vor Matching & 0,057 & 0,049 & 3,7 & 0,97 & 0,333 \\
\hline & Nach Matching & 0,061 & 0,047 & 6,2 & 1,10 & 0,273 \\
\hline \multirow[t]{2}{*}{ Ausbildung } & Vor Matching & 0,546 & 0,550 & $-0,8$ & $-0,21$ & 0,834 \\
\hline & Nach Matching & 0,535 & 0,550 & $-3,0$ & $-0,54$ & 0,587 \\
\hline \multirow[t]{2}{*}{ Fachschule } & Vor Matching & 0,068 & 0,083 & $-5,4$ & $-1,31$ & 0,190 \\
\hline & Nach Matching & 0,074 & 0,075 & $-0,3$ & $-0,05$ & 0,963 \\
\hline \multirow[t]{2}{*}{ (Fach-)Hochschulabschluss } & Vor Matching & 0,329 & 0,319 & 2,1 & 0,54 & 0,588 \\
\hline & Nach Matching & 0,330 & 0,328 & 0,4 & 0,07 & 0,942 \\
\hline \multirow[t]{2}{*}{ Alter $18-35$} & Vor Matching & 0,375 & 0,317 & 12,1 & 3,09 & 0,002 \\
\hline & Nach Matching & 0,352 & 0,339 & 2,8 & 0,50 & 0,617 \\
\hline \multirow[t]{2}{*}{ Alter $36-50$} & Vor Matching & 0,466 & 0,482 & $-3,4$ & $-0,84$ & 0,399 \\
\hline & Nach Matching & 0,489 & 0,482 & 1,3 & 0,24 & 0,813 \\
\hline \multirow[t]{2}{*}{ Alter 51-65 } & Vor Matching & 0,160 & 0,200 & $-10,6$ & $-2,57$ & 0,010 \\
\hline & Nach Matching & 0,159 & 0,179 & $-5,2$ & $-0,95$ & 0,342 \\
\hline \multirow[t]{2}{*}{ Geschlecht } & Vor Matching & 0,312 & 0,397 & $-17,8$ & $-4,40$ & 0,000 \\
\hline & Nach Matching & 0,295 & 0,308 & $-2,5$ & $-0,47$ & 0,640 \\
\hline \multirow[t]{2}{*}{ Verheiratet } & Vor Matching & 0,480 & 0,489 & $-1,9$ & $-0,47$ & 0,640 \\
\hline & Nach Matching & 0,492 & 0,487 & 1,0 & 0,17 & 0,863 \\
\hline \multirow[t]{2}{*}{ Kinder im $\mathrm{HH}$} & Vor Matching & 0,390 & 0,371 & 3,9 & 0,99 & 0,320 \\
\hline & Nach Matching & 0,403 & 0,384 & 3,8 & 0,68 & 0,499 \\
\hline \multirow[t]{2}{*}{ Staatsangehörigkeit Deutsch } & Vor Matching & 0,027 & 0,029 & $-1,3$ & $-0,32$ & 0,751 \\
\hline & Nach Matching & 0,028 & 0,029 & $-0,4$ & $-0,08$ & 0,940 \\
\hline \multirow[t]{2}{*}{ Verantwortungsvolle Tätigkeit } & Vor Matching & 0,832 & 0,671 & 37,9 & 8,77 & 0,000 \\
\hline & Nach Matching & 0,816 & 0,814 & 0,4 & 0,09 & 0,932 \\
\hline \multirow[t]{2}{*}{ Häufiges Arbeiten unter Leistungsdruck } & Vor Matching & 0,698 & 0,589 & 22,9 & 5,62 & 0,000 \\
\hline & Nach Matching & 0,682 & 0,662 & 4,3 & 0,78 & 0,434 \\
\hline \multirow[t]{2}{*}{ Wenig Handlungsspielraum vorhanden } & Vor Matching & 0,219 & 0,170 & 12,5 & 3,27 & 0,001 \\
\hline & Nach Matching & 0,197 & 0,197 & $-0,1$ & $-0,02$ & 0,981 \\
\hline \multirow[t]{2}{*}{ Auftreten von Monotonie } & Vor Matching & 0,430 & 0,418 & 2,4 & 0,61 & 0,544 \\
\hline & Nach Matching & 0,418 & 0,404 & 2,8 & 0,50 & 0,617 \\
\hline \multirow[t]{2}{*}{ Häufige neue Aufgaben } & Vor Matching & 0,132 & 0,089 & 13,9 & 3,77 & 0,000 \\
\hline & Nach Matching & 0,124 & 0,123 & 0,4 & 0,06 & 0,950 \\
\hline \multirow[t]{2}{*}{ Fachliche Überforderung } & Vor Matching & 0,040 & 0,035 & 2,5 & 0,63 & 0,526 \\
\hline & Nach Matching & 0,039 & 0,039 & $-0,0$ & $-0,00$ & 0,996 \\
\hline Fachliche Unterforderung & Vor Matching & 0,111 & 0,112 & $-0,2$ & $-0,05$ & 0,956 \\
\hline & Nach Matching & 0,112 & 0,142 & $-9,6$ & $-1,62$ & 0,106 \\
\hline Überforderung durch Arbeitspensum & Vor Matching & 0,261 & 0,178 & 20,0 & 5,33 & 0,000 \\
\hline & Nach Matching & 0,239 & 0,220 & 4,6 & 0,80 & 0,423 \\
\hline Unterforderung durch Arbeitspensum & Vor Matching & 0,043 & 0,045 & $-1,1$ & $-0,29$ & 0,775 \\
\hline & Nach Matching & 0,044 & 0,048 & $-1,8$ & $-0,32$ & 0,750 \\
\hline Häufiges Arbeiten im Stehen & Vor Matching & 0,580 & 0,386 & 39,5 & 10,01 & 0,000 \\
\hline & Nach Matching & 0,546 & 0,536 & 1,9 & 0,33 & 0,742 \\
\hline Häufiges Arbeiten im Sitzen & Vor Matching & 0,587 & 0,693 & $-22,2$ & $-5,74$ & 0,000 \\
\hline & Nach Matching & 0,601 & 0,631 & $-6,4$ & $-1,12$ & 0,264 \\
\hline
\end{tabular}


Tab. 8 (Fortsetzung)

\begin{tabular}{|c|c|c|c|c|c|c|}
\hline \multirow[t]{2}{*}{ Merkmal } & \multirow[t]{2}{*}{ Sample } & \multicolumn{2}{|l|}{ Mittelwert } & \multirow[t]{2}{*}{ SB } & \multirow[t]{2}{*}{$t$-Wert } & \multirow[t]{2}{*}{$p>|t|$} \\
\hline & & $\begin{array}{l}\text { Nachtschicht } \\
1 \text { bis } 4 \text { Nächte }\end{array}$ & Nicht-Schichtarbeit & & & \\
\hline \multirow[t]{2}{*}{ Häufiges Tragen der Lasten } & Vor Matching & 0,265 & 0,162 & 25,3 & 6,89 & 0,000 \\
\hline & Nach Matching & 0,230 & 0,214 & 3,8 & 0,67 & 0,502 \\
\hline \multirow[t]{2}{*}{ Häufiges Arbeiten in gebückter Haltung } & Vor Matching & 0,152 & 0,103 & 14,8 & 4,01 & 0,000 \\
\hline & Nach Matching & 0,134 & 0,133 & 0,1 & 0,02 & 0,986 \\
\hline \multirow[t]{2}{*}{ Häufiges Arbeiten mit Erschütterungen } & Vor Matching & 0,060 & 0,029 & 14,7 & 4,31 & 0,000 \\
\hline & Nach Matching & 0,061 & 0,044 & 8,7 & 1,43 & 0,153 \\
\hline \multirow[t]{2}{*}{ Häufiges Arbeiten unter Belastung der Atemwege } & Vor Matching & 0,339 & 0,244 & 21,1 & 5,54 & 0,000 \\
\hline & Nach Matching & 0,329 & 0,300 & 6,3 & 1,10 & 0,272 \\
\hline \multirow[t]{2}{*}{ Häufiges Arbeiten mit Gefahrenstoffe } & Vor Matching & 0,140 & 0,054 & 29,2 & 8,92 & 0,000 \\
\hline & Nach Matching & 0,096 & 0,110 & $-4,7$ & $-0,80$ & 0,421 \\
\hline \multirow[t]{2}{*}{ Häufiges Arbeiten bei Lärm } & Vor Matching & 0,278 & 0,181 & 23,2 & 6,24 & 0,000 \\
\hline & Nach Matching & 0,266 & 0,256 & 2,4 & 0,41 & 0,681 \\
\hline \multirow[t]{2}{*}{ Häufiges Arbeiten mit Mikroorganismen } & Vor Matching & 0,178 & 0,042 & 44,6 & 15,25 & 0,000 \\
\hline & Nach Matching & 0,124 & 0,111 & 4,5 & 0,75 & 0,451 \\
\hline \multirow[t]{2}{*}{ Dienstleistung (ohne öffentliche) } & Vor Matching & 0,311 & 0,399 & $-18,8$ & $-4,62$ & 0,000 \\
\hline & Nach Matching & 0,343 & 0,338 & 1,0 & 0,19 & 0,852 \\
\hline \multirow[t]{2}{*}{ Baugewerbe } & Vor Matching & 0,051 & 0,081 & $-12,1$ & $-2,81$ & 0,005 \\
\hline & Nach Matching & 0,057 & 0,042 & 5,9 & 1,20 & 0,231 \\
\hline \multirow[t]{2}{*}{ Gesundheitswesen } & Vor Matching & 0,225 & 0,072 & 44,1 & 13,86 & 0,000 \\
\hline & Nach Matching & 0,170 & 0,176 & $-1,7$ & $-0,28$ & 0,780 \\
\hline \multirow[t]{2}{*}{ Land und Forstwirtschaft } & Vor Matching & 0,007 & 0,017 & $-9,4$ & $-2,04$ & 0,041 \\
\hline & Nach Matching & 0,008 & 0,013 & $-4,9$ & $-0,93$ & 0,351 \\
\hline \multirow[t]{2}{*}{ Produzierendes Gewerbe } & Vor Matching & 0,228 & 0,300 & $-16,4$ & $-4,00$ & 0,000 \\
\hline & Nach Matching & 0,252 & 0,248 & 0,8 & 0,15 & 0,879 \\
\hline \multirow[t]{2}{*}{ Verkehr } & Vor Matching & 0,057 & 0,013 & 23,8 & 8,35 & 0,000 \\
\hline & Nach Matching & 0,047 & 0,052 & $-2,4$ & $-0,37$ & 0,714 \\
\hline \multirow[t]{2}{*}{ Öffentliche Dienstleistung } & Vor Matching & 0,121 & 0,116 & 1,6 & 0,41 & 0,679 \\
\hline & Nach Matching & 0,124 & 0,132 & $-2,3$ & $-0,40$ & 0,690 \\
\hline \multirow[t]{2}{*}{ Mittlere standardisierte Verzerrung } & Vor Matching & & 14,9 & & & \\
\hline & Nach Matching & & 3,0 & & & \\
\hline
\end{tabular}

Quelle: BIBB/BAUA-Erwerbstätigenbefragung 2006, alle Angaben gewichtet, eigene Berechnungen 
Tab. 9 Deskriptive Statistiken und Balancing der Variablen - Nachtschicht in 5 oder mehr Nächten im Monat und Vergleichsgruppe

\begin{tabular}{|c|c|c|c|c|c|c|}
\hline \multirow[t]{2}{*}{ Merkmal } & \multirow[t]{2}{*}{ Sample } & \multicolumn{2}{|l|}{ Mittelwert } & \multirow[t]{2}{*}{ SB } & \multirow[t]{2}{*}{$t$-Wert } & \multirow[t]{2}{*}{$p>|t|$} \\
\hline & & $\begin{array}{l}\text { Nachtschicht in } \\
>5 \text { Nächten }\end{array}$ & Nicht-Schichtarbeit & & & \\
\hline \multirow[t]{2}{*}{ Keine Ausbildung } & Vor Matching & 0,060 & 0,049 & 5,0 & 1,62 & 0,104 \\
\hline & Nach Matching & 0,059 & 0,073 & $-6,0$ & $-1,26$ & 0,208 \\
\hline \multirow[t]{2}{*}{ Ausbildung } & Vor Matching & 0,746 & 0,550 & 41,9 & 12,62 & 0,000 \\
\hline & Nach Matching & 0,732 & 0,757 & $-5,5$ & $-1,36$ & 0,174 \\
\hline \multirow[t]{2}{*}{ Fachschule } & Vor Matching & 0,050 & 0,083 & $-13,3$ & $-3,88$ & 0,000 \\
\hline & Nach Matching & 0,055 & 0,044 & 4,2 & 1,09 & 0,274 \\
\hline \multirow[t]{2}{*}{ (Fach-)Hochschulabschluss } & Vor Matching & 0,145 & 0,319 & $-42,2$ & $-12,17$ & 0,000 \\
\hline & Nach Matching & 0,154 & 0,139 & 5,0 & 1,63 & 0,104 \\
\hline \multirow[t]{2}{*}{ Alter $18-35$} & Vor Matching & 0,386 & 0,317 & 14,4 & 4,62 & 0,000 \\
\hline & Nach Matching & 0,382 & 0,351 & 6,5 & 1,48 & 0,138 \\
\hline \multirow[t]{2}{*}{ Alter $36-50$} & Vor Matching & 0,491 & 0,483 & 1,6 & 0,51 & 0,613 \\
\hline & Nach Matching & 0,485 & 0,501 & $-4,1$ & $-1,09$ & 0,273 \\
\hline \multirow[t]{2}{*}{ Alter 51-65 } & Vor Matching & 0,123 & 0,200 & $-21,0$ & $-6,20$ & 0,000 \\
\hline & Nach Matching & 0,133 & 0,118 & 3,9 & 1,00 & 0,319 \\
\hline \multirow[t]{2}{*}{ Geschlecht } & Vor Matching & 0,266 & 0,397 & $-28,0$ & $-8,52$ & 0,000 \\
\hline & Nach Matching & 0,281 & 0,284 & $-0,5$ & $-0,13$ & 0,896 \\
\hline \multirow[t]{2}{*}{ Verheiratet } & Vor Matching & 0,489 & 0,489 & $-0,1$ & $-0,03$ & 0,976 \\
\hline & Nach Matching & 0,478 & 0,489 & $-2,2$ & $-0,50$ & 0,617 \\
\hline \multirow[t]{2}{*}{ Kinder im $\mathrm{HH}$} & Vor Matching & 0,402 & 0,371 & 6,2 & 1,97 & 0,049 \\
\hline & Nach Matching & 0,391 & 0,387 & 0,8 & 0,17 & 0,861 \\
\hline \multirow[t]{2}{*}{ Staatsangehörigkeit Deutsch } & Vor Matching & 0,047 & 0,029 & 9,4 & 3,21 & 0,001 \\
\hline & Nach Matching & 0,044 & 0,039 & 3,0 & 0,66 & 0,509 \\
\hline \multirow[t]{2}{*}{ Verantwortungsvolle Tätigkeit } & Vor Matching & 0,671 & 0,671 & 0,0 & 0,01 & 0,993 \\
\hline & Nach Matching & 0,669 & 0,612 & 4,3 & 1,61 & 0,108 \\
\hline \multirow[t]{2}{*}{ Häufiges Arbeiten unter Leistungsdruck } & Vor Matching & 0,615 & 0,589 & 5,3 & 1,66 & 0,097 \\
\hline & Nach Matching & 0,611 & 0,571 & 5,2 & 1,63 & 0,102 \\
\hline \multirow[t]{2}{*}{ Wenig Handlungsspielraum vorhanden } & Vor Matching & 0,359 & 0,170 & 43,8 & 15,09 & 0,000 \\
\hline & Nach Matching & 0,317 & 0,320 & $-0,5$ & $-0,12$ & 0,907 \\
\hline \multirow[t]{2}{*}{ Auftreten von Monotonie } & Vor Matching & 0,605 & 0,418 & 38,1 & 11,94 & 0,000 \\
\hline & Nach Matching & 0,584 & 0,616 & $-6,6$ & $-1,51$ & 0,130 \\
\hline \multirow[t]{2}{*}{ Häufige neue Aufgaben } & Vor Matching & 0,104 & 0,089 & 5,2 & 1,69 & 0,090 \\
\hline & Nach Matching & 0,105 & 0,093 & 4,4 & 1,00 & 0,318 \\
\hline \multirow[t]{2}{*}{ Fachliche Überforderung } & Vor Matching & 0,057 & 0,035 & 10,6 & 3,62 & 0,000 \\
\hline & Nach Matching & 0,054 & 0,046 & 3,7 & 0,83 & 0,407 \\
\hline Fachliche Unterforderung & Vor Matching & 0,175 & 0,112 & 18,2 & 6,15 & 0,000 \\
\hline & Nach Matching & 0,164 & 0,170 & $-1,8$ & $-0,38$ & 0,703 \\
\hline Überforderung durch Arbeitspensum & Vor Matching & 0,211 & 0,178 & 8,4 & 2,69 & 0,007 \\
\hline & Nach Matching & 0,212 & 0,187 & 6,2 & 1,41 & 0,159 \\
\hline Unterforderung durch Arbeitspensum & Vor Matching & 0,066 & 0,046 & 9,1 & 3,06 & 0,002 \\
\hline & Nach Matching & 0,065 & 0,060 & 2,1 & 0,45 & 0,650 \\
\hline Häufiges Arbeiten im Stehen & Vor Matching & 0,770 & 0,386 & 84,3 & 25,29 & 0,000 \\
\hline & Nach Matching & 0,758 & 0,745 & 2,9 & 0,70 & 0,484 \\
\hline Häufiges Arbeiten im Sitzen & Vor Matching & 0,374 & 0,693 & $-67,4$ & $-21,57$ & 0,000 \\
\hline & Nach Matching & 0,385 & 0,397 & $-2,6$ & $-0,57$ & 0,566 \\
\hline
\end{tabular}


Tab. 9 (Fortsetzung)

\begin{tabular}{|c|c|c|c|c|c|c|}
\hline \multirow[t]{2}{*}{ Merkmal } & \multirow[t]{2}{*}{ Sample } & \multicolumn{2}{|l|}{ Mittelwert } & \multirow[t]{2}{*}{ SB } & \multirow[t]{2}{*}{$t$-Wert } & \multirow[t]{2}{*}{$p>|t|$} \\
\hline & & $\begin{array}{l}\text { Nachtschicht in } \\
>5 \text { Nächten }\end{array}$ & Nicht-Schichtarbeit & & & \\
\hline \multirow[t]{2}{*}{ Häufiges Tragen der Lasten } & Vor Matching & 0,324 & 0,162 & 38,4 & 13,20 & 0,000 \\
\hline & Nach Matching & 0,315 & 0,304 & 2,6 & 0,54 & 0,587 \\
\hline \multirow[t]{2}{*}{ Häufiges Arbeiten in gebückter Haltung } & Vor Matching & 0,158 & 0,103 & 16,3 & 5,46 & 0,000 \\
\hline & Nach Matching & 0,155 & 0,142 & 3,9 & 0,84 & 0,401 \\
\hline \multirow[t]{2}{*}{ Häufiges Arbeiten mit Erschütterungen } & Vor Matching & 0,109 & 0,029 & 31,6 & 12,61 & 0,000 \\
\hline & Nach Matching & 0,078 & 0,078 & 0,1 & 0,02 & 0,987 \\
\hline \multirow[t]{2}{*}{ Häufiges Arbeiten unter Belastung der Atemwege } & Vor Matching & 0,569 & 0,243 & 70,3 & 23,30 & 0,000 \\
\hline & Nach Matching & 0,534 & 0,539 & $-1,1$ & $-0,24$ & 0,807 \\
\hline \multirow[t]{2}{*}{ Häufiges Arbeiten mit Gefahrenstoffe } & Vor Matching & 0,156 & 0,054 & 33,6 & 12,68 & 0,000 \\
\hline & Nach Matching & 0,137 & 0,151 & $-4,8$ & $-0,96$ & 0,337 \\
\hline \multirow[t]{2}{*}{ Häufiges Arbeiten bei Lärm } & Vor Matching & 0,442 & 0,181 & 58,8 & 20,34 & 0,000 \\
\hline & Nach Matching & 0,407 & 0,406 & 0,4 & 0,08 & 0,938 \\
\hline \multirow[t]{2}{*}{ Häufiges Arbeiten mit Mikroorganismen } & Vor Matching & 0,169 & 0,042 & 42,2 & 16,95 & 0,000 \\
\hline & Nach Matching & 0,163 & 0,123 & 6,9 & 1,61 & 0,107 \\
\hline \multirow[t]{2}{*}{ Dienstleistung (ohne öffentliche) } & Vor Matching & 0,183 & 0,400 & $-49,1$ & $-14,33$ & 0,000 \\
\hline & Nach Matching & 0,201 & 0,235 & $-6,7$ & $-1,59$ & 0,112 \\
\hline \multirow[t]{2}{*}{ Baugewerbe } & Vor Matching & 0,013 & 0,081 & $-32,7$ & $-8,43$ & 0,000 \\
\hline & Nach Matching & 0,014 & 0,011 & 1,4 & 0,58 & 0,561 \\
\hline \multirow[t]{2}{*}{ Gesundheitswesen } & Vor Matching & 0,178 & 0,072 & 32,5 & 11,88 & 0,000 \\
\hline & Nach Matching & 0,183 & 0,155 & 7,4 & 1,58 & 0,114 \\
\hline \multirow[t]{2}{*}{ Land und Forstwirtschaft } & Vor Matching & 0,020 & 0,017 & 1,7 & 0,53 & 0,594 \\
\hline & Nach Matching & 0,021 & 0,023 & $-1,8$ & $-0,38$ & 0,707 \\
\hline \multirow[t]{2}{*}{ Produzierendes Gewerbe } & Vor Matching & 0,447 & 0,300 & 30,7 & 9,94 & 0,000 \\
\hline & Nach Matching & 0,440 & 0,445 & $-1,1$ & $-0,25$ & 0,800 \\
\hline \multirow[t]{2}{*}{ Verkehr } & Vor Matching & 0,068 & 0,013 & 27,7 & 11,80 & 0,000 \\
\hline & Nach Matching & 0,044 & 0,052 & $-4,0$ & $-0,85$ & 0,396 \\
\hline \multirow[t]{2}{*}{ Öffentliche Dienstleistung } & Vor Matching & 0,092 & 0,116 & $-7,9$ & $-2,41$ & 0,016 \\
\hline & Nach Matching & 0,098 & 0,079 & 6,4 & 1,58 & 0,115 \\
\hline \multirow[t]{2}{*}{ Mittlere standardisierte Verzerrung } & Vor Matching & & 26,3 & & & \\
\hline & Nach Matching & & 4,2 & & & \\
\hline
\end{tabular}

Quelle: BIBB/BAUA-Erwerbstätigenbefragung 2006, alle Angaben gewichtet, eigene Berechnungen 


\section{Literatur}

Agervold, M.: Shiftwork: a critical review. Scand. J. Psychol. 17, 181188 (1976)

Åkerstedt, T.: Inversion of the sleep wakefulness pattern: effects on circadian variations in psychophysiological activation. Ergonomics 20, 459-474 (1977)

Åkerstedt, T.: Shift work and disturbed sleep/wakefulness. Occup. Med. (Oxf.) 53, 89-94 (2003)

Åkerstedt, T., Gillberg, M.: Sleep disturbances and shift work. In: Reinberg, A., Vieux, N., Andlauer, P. (Hrsg.) Night and Shift Work: Biological and Social Aspects: Proceedings of the Vth International Symposium on Night and Shift Work, S. 127-138. Pergamon, Oxford (1981)

Ahasan, R., Lewko, J., Campbell, D., Salmoni, A.: Adaptation to night shifts and synchronisation processes of night workers. J. Physiol. Anthropol. Appl. Hum. Sci. 20, 215-226 (2001)

Amelsvoort, L.G., Van, Schouten, E., Kok, F.J.: Impact of one year of shift work on cardiovascular disease risk factors. J. Occup. Environ. Med. 46, 699-706 (2004)

Andersen, J.E.: Three-Shift Work. A Socio-Medical Survey, vols. I and II. Teknisk Forlag, Copenhagen (1970)

Angersbach, D., Knauth, P., Loskant, H., Karvonen, M.J., Undeutsch, K., Rutenfranz, J.: A retrospective cohort study comparing complaints and diseases on day and shift workers. Int. Arch. Occup. Environ. Health 45, 127-140 (1980)

Badura, B., Feuerstein, G.: Systemgestaltung im Gesundheitswesen: Zur Versorgungskrise der Hochtechnisierten Medizin und den Möglichkeiten ihrer Bewältigung. Juventa, München (1996)

BAG: Urteil des Bundesarbeitsgericht vom 20. Juni 1990, 4 AZR 5/90. Erfurt (1990)

Baillargeon, J.: Characteristics of the healthy worker effect. Occup. Med. (Oxf.) 16, 359-366 (2001)

Becker, S., Ichino, A.: Estimation of average treatment effects based on propensity scores. Stata J. 4, 358-377 (2002)

Beermann, B.: Nacht- und Schichtarbeit. In: Badura, B., Schröder, H., Klose, J., Macco, K. (Hrsg.) Fehlzeiten-Report 2009, S. 71-82. Springer, Heidelberg (2010)

BIBB/BAuA: Erwerbstätigenbefragung 2005/2006 Arbeit und Beruf im Wandel, Erwerb und Verwertung beruflicher Qualifikationen. Erhebungsinstrument. Fragebogenmaster für die CATI-Programmierung incl. Variablenkennung. Bonn, Dortmund (2006)

Bøggild, H., Knutsson, A.: Shift work, risk factors and cardiovascular disease. Scand. J. Work, Environ. \& Health 25, 85-99 (1999)

Bøggild, H., Suadicani, P., Hein, H.O., Gyntelberg, F.: Shift work, social class, and ischaemic heart disease in middle aged and elderly men; a 22 year follow up in the Copenhagen male study. Occup. Environ. Med. (Lond.) 56, 640-645 (1999)

Bøggild, H., Burr, H., Tüchsen, F., Jeppesen, H.J.: Work environment of Danish shift and day workers. Scand. J. Work, Environ. \& Health 27, 97-105 (2001)

Bohle, P., Tilley, A.J.: The impact of night work on psychological wellbeing. Ergonomics 32, 375-376 (1989)

Caliendo, M., Kopeinig, S.: Some practical guidance for the implementation of propensity score matching. J. Econ. Surv. 22, 31-72 (2008)

Caruso, C.C., Lusk, S.L., Gillespie, B.W.: Relationship of work schedules to gastrointestinal diagnoses, symptoms, and medication use in auto factory workers. Am. J. Ind. Med. 46, 586-598 (2004)

Costa, G.: The impact of shift and night work on health. Appl. Ergon. 27, 9-16 (1996)

Costa, G.: Shiftwork and health: the heritage of the twentieth century. In: Hornberger, S., Knauth, P., Costa, G., Folkard, S. (Hrsg.) Shiftwork in the 21st Century, S. 155-160. Peter Lang, Frankfurt am Main (2000)
Costa, G.: Multidimensional aspects related to shiftworkers' health and well-being. Rev. Panam. Salud Pública 38, 86-91 (2004)

Costa, G., Haus, E., Stevens, R.: Shift work and cancerconsiderations on rationale, mechanisms, and epidemiology. Scand. J. Work, Environ. \& Health 36, 163-179 (2010)

Dehejia, R., Wahba, S.: Propensity score-matching methods for nonexperimental causal studies. Rev. Econ. Stat. 84, 151-161 (2002)

Estryn-Behar, M., Gadbois, C., Peigne, E., Masson, A., Le Gall, V.: Impact of nightshifts on male and female hospital staff. In: Costa, C., Cesana, G.C., Kogi, K., Wedderburn, A.A.I. (Hrsg.) Shiftwork: Health, Sleep and Performance, S. 89-94. Peter Lang, Frankfurt am Main (1990)

Folkard, S., Barton, J.: Does the "forbidden zone" for sleep onset influence morning shift sleep duration? Ergonomics 36(1-3), 85-91 (1993)

Folkard, S., Tucker, P.: Shift work, safety and productivity. Occup. Med. (Oxf.) 53, 95-101 (2003)

Frost, P., Kolstad, H.A., Bonde, J.P.: Shift work and the risk of ischemic heart disease - a systematic review of the epidemiologic evidence. Scand. J. Work, Environ. \& Health 35, 163-179 (2009)

Gadbois, C.: Shiftwork and Health. European Foundation for the Improvement of Living and Working Conditions, Dublin (1996)

Gangl, M., DiPrete, T.: Kausalanalyse durch Matchingverfahren. In: Diekmann, A. (Hrsg.) Methoden der Sozialforschung. Sonderheft der Kölner Zeitschrift für Soziologie und Sozialpsychologie, S. 396-420 (2006)

Hacker, W.: Allgemeine Arbeitspsychologie: Psychische Regulation von Wissens-, Denk- und körperlicher Arbeit. Huber, Bern (2005)

Hajak, G., Zulley, J.: Schlafstörungen bei Schichtarbeit - Wenn die Arbeit mit der inneren Uhr kollidiert. Psychoneuro 34, 70-77 (2008)

Hall, A., et al.: BIBB/BAuA-Erwerbstätigenbefragung 2006: Arbeit und Beruf Im Wandel, Erwerb und Verwertung beruflicher Qualifikationen - Abschlussbericht. Bundesinstitut für Berufsbildung, Bonn (2010)

Hartmann, J.: BIBB/BAuA-Erwerbstätigenbefragung 2005/2006. Feldbericht (TNS Infratest Sozialforschung). München (2006a)

Hartmann, J.: BIBB/BAuA-Erwerbstätigenbefragung 2005/2006. Strukturkontrolle, Steuerung und Gewichtung der Stichprobe (TNS Infratest Sozialforschung). München (2006b)

Holland, P.W.: Statistics and causal inference. J. Am. Stat. Assoc. 81, 945-960 (1986)

Hublin, C., Partinen, M., Koskenvuo, K., Silventoinen, K., Koskenvuo, M., Kaprio, J.: Shift-work and cardiovascular disease: a population-based 22-year follow-up study. Eur. J. Epidemiol. 25, 315-323 (2010)

IARC: IARC Monographs Programme Finds Cancer Hazards Associated with Shiftwork, Painting and Firefighting. Press release No. 180, 5 December 2007 World Health Organization. International Agency for Research on Cancer, Lyon (2007)

Jamal, M.: Burnout, stress and health of employees on non-standard work schedules: a study of Canadian workers. Stress Health 20, 113-119 (2004)

Jansen, N.W., Kant, I., Nijhuis, F.J., Swaen, G.M., Kristensen, T.S.: Impact of worktime arrangements on work-home interference among Dutch employees. Scand. J. Work, Environ. \& Health 30, 139-148 (2004)

Janßen, C.: Soziale Schicht und 'Gesundheitliche Kontrollüberzeugungen'. In: Mielck, A., Bloomfield, K. (Hrsg.) SozialEpidemiologie, S. 184-195. Juventa, Weinheim (2001)

Jungbauer-Gans, M.: Soziale und kulturelle Einflüsse auf Krankheit und Gesundheit: Theoretische Überlegungen. Köln. Z. Soziol. Soz.psychol. 46, 86-108 (2006)

Karasek, R.A., Theorell, T.: Healthy Work: Stress, Productivity and the Reconstruction of Working Life. Basic Books, New York (1990)

Karlsson, B., Knutsson, A., Lindahl, B.: Is there an association between shift work and having a metabolic syndrome? Results from 
a population based study of 27.485 people. Occup. Environ. Med. (Lond.) 58, 747-752 (2001)

Kawachi, I., Colditz, G.A., Stampfer, M.J., Willett, W.C., Manson, J.E., et al.: Prospective study of shift work and risk of coronary heart disease in women. Circulation 92, 3178-3182 (1995)

Kecklund, G., Åkerstedt, T.: Effects of timing of shifts on sleepiness and sleep duration. J. Sleep Res. 4(2), 47-50 (1995)

Knauth, P.: Schichtarbeit. In: Rohmert, W., Rutenfranz, J. (Hrsg.) Praktische Arbeitsphysiologie, S. 368-375. Thieme, Stuttgart (1983)

Knauth, P.: Schlafstörungen bei Schichtarbeitern. In: Faust, V. (Hrsg.) Schlafstörungen. Häufigkeit - Ursachen - Schlafmittel - nichtmedikamentöse Schlafhilfen, S. 90-93. Hippokrates, Stuttgart (1985)

Knauth, P.: The design of shift systems. Ergonomics 36(1-3), 15-28 (1993)

Knauth, P., Rutenfranz, J.: Duration of sleep and type of shift work. In: Reinberg, A., Vieux, N., Andlauer, P. (Hrsg.) Night and Shift Work: Biological and Social Aspects: Proceedings of the Vth International Symposium on Night and Shift Work, S. 161-168. Pergamon, Oxford (1981)

Knauth, P., Landau, K., Dröge, C., Schwitteck, M., Widynski, M., Rutenfranz, J.: Duration of sleep depending on the type of shift work. Int. Arch. Occup. Environ. Health 46, 167-177 (1980)

Knauth, P., Kiesswetter, E., Ottoman, W., Karvonen, M.J., Rutenfranz, J.: Time budget studies of policemen in weekly or swiftly rotating shift systems. Appl. Ergon. 14, 247-252 (1983)

Knutsson, A.: Health disorders of shift workers. Occup. Med. (Oxf.) 53, 103-108 (2003)

Kolstad, H.A.: Nightshift work and risk of breast cancer and other cancers - a critical review of the epidemiologic evidence. Scand. J. Work, Environ. \& Health 34, 5-22 (2008)

Kolstad, H.A., Erlandsen, M., Frost, P., Bonde, J.P.: Should we warn against night shifts to prevent breast cancer? Occup. Environ. Med. (Lond.) 67, 797 (2010)

Leuven, E., Sianesi, B.: PSMATCH2: stata module to perform full Mahalanobis and propensity score matching, common support graphing, and covariate imbalance testing. Version 4.0.5 (2003). http://ideas.repec.org/c/boc/bocode/s432001.html. Eingesehen am 20. Juni 2012

Mirowsky, J., Ross, C.: Social Causes of Psychological Distress. Aldine Transaction, New Brunswick (2003)

Paridon, H., Ernst, S., Harth, V., Nickel, P., Nold, A., Pallapies, D.: Schichtarbeit. Rechtslage, gesundheitliche Risiken und Präventionsmöglichkeiten. DGUV report 1/2012 (2012)

Pesch, B., Harth, V., Rabstein, S., Baisch, C., Schiffermann, M., Pallapies, D., Bonberg, N., Heinze, E., Spickenheuer, A., Justenhoven, C., Brauch, H., Hamann, U., Ko, Y.D., Straif, K., Brüning, T.: Night work and breast cancer-results from the German GENICA study. Scand. J. Work, Environ. \& Health 36, 134-141 (2010)

Pisarski, A., Bohle, P., Callan, V.J.: Extended shifts in ambulance work: influences on health. Stress Health 18, 119-126 (2002)

Pronk, A., Ji, B.T., Shu, X.O., et al.: Night-shift work and breast cancer risk in a cohort of Chinese women. Am. J. Epidemiol. 171, 953959 (2010)

Pukkala, E., Härmä, M.: Does shift work cause cancer? Scand. J. Work, Environ. \& Health 33, 321-323 (2007)

Richter, K.D., Acker, J., Scholz, F., Niklewski, G.: Health promotion and work: prevention of shift work disorders in companies. EPMA Journal 1, 611-618 (2010)

Rosenbaum, P.R., Rubin, D.B.: The central role of the propensity score in observational studies for causal effects. Biometrika 70, 41-55 (1983)

Sakata, K., Suwazono, Y., Harada, H., Okubo, Y., Kobayashi, E., Nogawa, K.: The relationship between shift work and the onset of hypertension in male Japanese workers. J. Occup. Environ. Med. 45, 1002-1006 (2003)
Schernhammer, E.S., Kroenke, C.H., Laden, F., Hankinson, S.E.: Night work and risk of breast cancer. Epidemiology 17, 108-111 (2006)

Schlottfeldt, C.: Arbeitszeitrecht. In: Ignor, A., Rixen, S. (Hrsg.) Handbuch Arbeitsstrafrecht: Personalverantwortung als Strafbarkeitsrisiko, S. 469-540. Boorberg, Stuttgart (2008)

Segawa, K., Nakazawa, S., Tsukamoto, Y., Kurita, Y., Goto, H., et al.: Peptic ulcer is prevalent among shift workers. Dig. Dis. Sci. 32, 449-453 (1987)

Seifert, H.: Zeit für neue Arbeitszeiten. WSI-Mitt. 58, 478-483 (2005)

Siegrist, J., Dragano, N.: Berufliche Belastungen und Gesundheit. Köln. Z. Soziol. Soz.psychol., Sonderh. 46, 109-124 (2006)

Straif, K., Baan, R., Grosse, Y., et al.: Carcinogenicity of shift-work, painting, and fire-fighting. Lancet Oncol. 8, 1065-1066 (2007)

Tenkanen, L., Sjöblom, T., Kalimo, R., Alikoski, T., Härmä, M.: Shiftwork, occupation and coronary heart disease over 6 years of follow-up in the Helsinki heart study. Scand. J. Work, Environ. \& Health 23, 257-265 (1997)

Tilley, A.J., Wilkinson, R.T., Drud, M.: Night and day shifts compared in terms of the quality and quantity of sleep recorded in the home and performance measured at work: a pilot study. In: Reinberg, A., Vieux, N., Andlauer, P. (Hrsg.) Night and Shift Work: Biological and Social Aspects, Proceedings of the Vth International Symposium on Night and Shift Work, S. 187-196. Pergamon, Oxford (1981)

Thomas, C., Power, C.: Shift work and risk factors for cardiovascular disease: a study at age 45 years in the 1958 British birth cohort. Eur. J. Epidemiol. 25, 305-314 (2010)

Urban, D., Mayerl, J.: Regressionsanalyse: Theorie, Technik und Anwendung. VS Verlag, Wiesbaden (2006)

Van Cauter, E., Sturis, J., Byrne, M.M., Blackman, J.D., Leproult, R., et al.: Demonstration of rapid light-induced advances and delays of the human circadian clock using hormonal phase markers. Am. J. Physiol. 266, E953-E963 (1994)

Wedderburn, A.A.I.: Studies of Shiftwork in the Steel Industry. Report to the BSC Fellowships Committee. British Steel Corporation, London (1975)

Wedderburn, A.A.I.: Shiftwork and Health. BEST European Studies on Time. European Foundation for the Improvement of Living and Working Conditions, Dublin (2000)

Olaf Struck ist Professor für Arbeitswissenschaft an der OttoFriedrich-Universität Bamberg. Hier leitet er u.a. den Bereich Lernumwelten im Nationalen Bildungspanel (NEPS) sowie den Bereich Human Capital, Labour Markets and Demographic Structures der Bamberg Graduate School of Social Sciences (BAGSS). Zudem ist er im Vorstand der deutschen Gesellschaft für Arbeitsmarktforschung. Forschungsinteressen: empirische Arbeitsmarkt-, Organisations- und Bildungsforschung.

Matthias Dütsch absolvierte ein Studium der Sozialwissenschaften an der Friedrich-Alexander-Universität Erlangen-Nürnberg, welches er 2008 als Diplom Sozialwirt abschloss. Seit 2009 ist er wissenschaftlicher Mitarbeiter an der Professur für Arbeitswissenschaft der OttoFriedrich-Universität Bamberg. Seine aktuellen Forschungsinteressen in der empirischen Arbeitsmarktforschung sind: atypische Beschäftigung, betriebliche Beschäftigung und Beschäftigungssysteme sowie Erwerbsverläufe. 
Verena Liebig absolvierte ihr Psychologiestudium mit dem Schwerpunkt Arbeits- \& Organisationspsychologie an der Friedrich-AlexanderUniversität Erlangen-Nürnberg sowie an der University of St. Andrews in Schottland. Nachdem sie 2004 als Diplom-Psychologin abschloss, arbeitete sie 6 Jahre im Personalmanagement eines großen deutschen Handelskonzerns. Seit 2010 ist sie als Lehrkraft für besondere Aufgaben und wissenschaftliche Mitarbeiterin an der Professur für Arbeitswissenschaft der Otto-Friedrich-Universität Bamberg tätig.
Angelina Springer studierte von 2006 bis 2012 Soziologie an der Universität Bamberg mit dem Schwerpunkt „Methoden der empirischen Sozialforschung“ (Diplom). Seit Juni 2012 ist sie wissenschaftliche Mitarbeiterin am IAB. Zu ihren Forschungsinteressen gehört Qualität der Arbeit und Sozialpolitik. 